\title{
A computationally inexpensive model for estimating dimensional measurement uncertainty due to X-ray computed tomography instrument misalignments
}

\author{
${ }^{1}$ Mechanical Engineering Department, KU Leuven (Belgium) \\ 2 Engineering Measurement Group, National Physical Laboratory, Teddington (UK) \\ ${ }^{3}$ Institute of Data Processing and Electronics, Karlsruhe Institute of Technology, Karlsruhe (Germany)
}

\begin{abstract}
The recent emergence of advanced manufacturing techniques such as additive manufacturing and an increased demand on the integrity of components have motivated research on the application of X-ray computed tomography (CT) for dimensional quality control. While CT has shown significant empirical potential for this purpose, there is a need for metrological research to accelerate the acceptance of CT as a measuring instrument. The accuracy in CT-based measurements is vulnerable to the instrument geometrical configuration during data acquisition, namely the relative position and orientation of Xray source, rotation stage, and detector. Consistency between the actual instrument geometry and the corresponding parameters used in the reconstruction algorithm is critical. Currently available procedures provide users with only estimates of geometrical parameters. Quantification and propagation of uncertainty in the measured geometrical parameters must be considered to provide a complete uncertainty analysis and to establish confidence intervals for CT dimensional measurements. In this paper, we propose a computationally inexpensive model to approximate the influence of errors in CT geometrical parameters on dimensional measurement results. We use surface points extracted from a computer-aided design (CAD) model to model discrepancies in the radiographic image coordinates assigned to the projected edges between an aligned system and a system with misalignments. The efficacy of the proposed method was confirmed on simulated and experimental data in the presence of various geometrical uncertainty contributors.
\end{abstract}




\section{Introduction}

X-ray computed tomography (CT) is an imaging technique that employs penetrating electromagnetic radiation and dedicated mathematical algorithms to visualize, analyze and quantify both external and internal structures of the measured object in a non-destructive manner. The potential of CT as a multipurpose and effective measuring technology is widely recognized. However, the application of CT to metrologically rigorous tasks, such as dimensional quality control of manufactured products, demands considerable research efforts. The formal estimation and expression of measurement uncertainty is fundamental for acceptance of CT as a measuring instrument. This study applies to typical industrial cone-beam CT systems.

The tomographic reconstruction step in CT generates a three-dimensional attenuation map (volume) of the measured object from a series of two-dimensional projections (radiographs) acquired at different perspectives. Attenuation maps consist of three-dimensional arrays of voxels (threedimensional equivalent of pixels), each having a grey value intensity within a specified range corresponding to the X-ray attenuation at the respective unit volume. While these 3D volumes provide users with visual information about the measured object, additional steps are required to extract dimensional information. The 3D attenuation map is converted to a surface model by imageprocessing operations such as edge-based segmentation, in which material interfaces are specified by a significant transition between high and low intensities (large image gradients). Surface sampling can then be applied to the surface model to extract a point cloud of surface coordinates.

Accurate CT reconstruction demands that the geometrical configuration during data acquisition, namely relative position and orientation of X-ray source, rotation stage, and detector, be consistent with the corresponding parameters used in the reconstruction algorithm [1]. For brevity, we use the word misalignment to refer to any type of inconsistency in geometrical parameters. Currently, available procedures provide users with only estimates of geometrical parameters. Quantification and propagation of uncertainty in the measured geometrical parameters must be considered to provide a complete uncertainty analysis and to establish confidence intervals for CT dimensional measurements.

In previous work [2], we have shown that geometrical misalignments lead to inconsistent radiographic data, which results in various visual artifacts in the reconstructed volume and consequent errors in dimensional measurements. Due to the complex relationship between the presence of geometrical misalignments and errors in dimensional measurements, analytical expressions linking geometrical parameters to dimensional measurements are currently not available. As a result, the conventional GUM method of uncertainty determination [3], which is based on the presence of an analytical measurement model to propagate uncertainty components, cannot be applied [4]. An alternative to the GUM method is based on Monte Carlo variation of input parameters in a simulated CT measurement. However, the computational load associated with simulation of the CT measurement procedure, i.e. simulation of radiographic image acquisition, tomographic reconstruction of the CT volume, and surface segmentation, prohibits its practical application to real measurement tasks [5], [6]. These limitations motivate us to find an alternative method for quantifying the influence of geometrical misalignments on dimensional measurements. A critical condition for this alternative method is that it is not computationally intensive, yet captures error behaviors and their influence on dimensional measurements.

The dimensional extent of an object can be defined by its surface. The inconsistency between the assumed geometry used in the back projection step of the reconstruction algorithm and the actual system geometry will result in errors in position of the segmented surface and consequently in errors in dimensional measurements. In this study, we introduce a new method for modelling surface coordinate errors due to geometrical misalignments without the need to perform a full CT simulation routine.

The proposed method is rooted in approaches for model-based reconstruction [7], [8] and reconstruction of shapes from silhouettes [9]. These previous approaches aim to reconstruct object features from incomplete and noisy data. In our proposed method, discrepancies in the radiographic 
image coordinates assigned to the projected edges between an aligned system and a system with misalignments are modelled. Our goal is to utilize the modelled radiographic discrepancies to quantify dimensional errors in the tomographically reconstructed edges. In a previous publication [2], distortions in the radiographic data due to detector angular misalignments were evaluated by way of a forward projection model. In this study, we extend our previous work and propose a method to propagate known misalignments to surface coordinate errors in the measurement volume. In previous work [2], only angular misalignments of the detector are considered. Here, we model the effects from all seven parameters that define the perspective projection geometry in a cone-beam CT system [10], [11]. The proposed algorithm is designed to provide computational benefits in the assessment of coordinate measurement uncertainty when compared to a full Monte Carlo simulation. A comparison of the algorithm output to observed surface deviations from simulated data and experimentally acquired tomographic data provide a proof of concept for the future application of the proposed method for assessing the contribution from instrument geometrical misalignments to taskspecific measurement uncertainty.

The rest of the paper is organized as follows. We start by introducing the notation used throughout the paper and summarizing the underlying assumptions within our work in sections 2 and 3, respectively. In section 4, the geometry of a typical cone-beam X-ray CT system with a circular scanning trajectory is described and the influence of geometrical misalignments on the measurement volume is discussed. In section 5, we introduce the geometrical error model. Sections 6 and 7 demonstrate the application of the proposed method to propagation of error in geometrical parameters to error in dimensional measurements with simulated CT datasets and experimentally acquired datasets, respectively. Monte Carlo procedure consists of repeated evaluations of a known model, each time varying the input values. Input parameters sampled from corresponding probability distributions. Consequently, for a single Monte Carlo run, the model is evaluated with exact values.

\section{Notation}

We use italic lowercase letters $a$ to denote scalars and bold italic letters $\boldsymbol{a}$ to denote vectors; vectors are always column vectors. Bold italicized uppercase letters $\boldsymbol{A}$ correspond to matrices. Non-italicized uppercase letters A are used for sets and surfaces. We use the formalism of homogeneous coordinates to describe transformations. A four-component column vector $\boldsymbol{c}=\left(a c_{x}, a c_{y}, a c_{z}, a\right)$ is the homogeneous equivalent of vector $\boldsymbol{c}=\left(c_{x}, c_{y}, c_{z}\right)$, where $a$ is the homogeneous scaling factor. We make no distinction between homogeneous or inhomogeneous vectors or matrices. Therefore, a threedimensional point coordinate can be defined as a $3 \times 1$ or $4 \times 1$ vector, depending on the context. The Euclidean scalar-product and cross-product of two vectors are denoted by $\langle\boldsymbol{a}, \boldsymbol{b}\rangle$ and $(\boldsymbol{a} \times \boldsymbol{b})$, respectively. $\|\boldsymbol{a}\|$ is the corresponding Euclidean vector norm. Vector transpose is denoted by superscript $T$, for example the vector transpose of $\boldsymbol{a}$ is given by $\boldsymbol{a}^{T} .|a|$ is the absolute value of the scalar $a$.

\section{Assumptions}

In this study, we focus only on the effects of geometrical misalignments, which are estimated in a separate procedure [10], [11]. We do not consider any other influence factors, such as noise, beamhardening, discretization error, cone-beam artifact, blurring, undersampling, etc. The X-ray focal spot is modelled as an infinitesimally small point source, the position of which is fixed (i.e. no focal spot drift). Rotary stage error motions are also not considered. The detector and measurement volume are not discretized into 2D pixel and 3D voxel grids, respectively. Therefore, the CT imaging system is treated as continuous space. 


\section{Cone-beam geometry}

The geometry of a cone-beam CT system is described as the relative position and orientation of the three major instrument components: X-ray focal spot, axis of object rotation, and flat-panel detector. In this section we parameterize the geometry of a typical cone-beam CT system with circular scanning trajectory and discuss the influence of geometrical misalignments on reconstruction results.

\subsection{Influence of geometrical misalignments}

The acquisition of radiographs for cone-beam CT consists of forward projecting a cone-shaped X-ray beam from the source focal spot, through the measurement volume, and onto a two-dimensional flatpanel detector. The cone-beam can be modelled as a series of straight line trajectories (rays) from the source point to the detector plane. As the X-rays traverse the measurement volume, they are attenuated by matter along their respective trajectories. The intensity of X-rays along each path is measured by the detector element onto which they are incident. Radiographic intensity images are acquired from different angular positions of the measured object and stored into an acquisition dataset.

CT reconstruction consists of generating a three-dimensional attenuation map of the measurement volume discretized onto a three-dimensional Cartesian lattice from the set of acquired twodimensional radiographs. One of the most commonly used tomographic reconstruction methods is the Feldkamp-Davis-Kress (FDK) algorithm, which is an extension of the single-slice filtered backprojection algorithm to cone-beam geometries. The popularity of the FDK algorithm is in part due to its computational benefits [12]. The attenuation grey value for each voxel in the measurement volume is calculated from the collection of measured pixel intensities corresponding to the X-ray trajectories intersecting that voxel at all rotation positions. The path traversed by each X-ray trajectory through the measurement volume is dependent on the position and orientation of the voxel space relative to the X-ray source spot and the corresponding pixel from which the trajectory is back projected for all rotation positions of the measurement volume. Accurate back projection of measured X-ray intensities therefore depends on accurate knowledge of the CT geometrical parameters. Discrepancies between actual acquisition geometry and the assumed geometry in the tomographic back projection step will generate erroneous volumetric data. This concept is illustrated for the single-slice reconstruction of a sphere from data acquired with an ideally aligned CT system and again with a misaligned CT system. For simplicity of illustration, parallel-beam geometry is used. Figure 1a illustrates the back projection of the imaged disc (single slice of a sphere) from an aligned system for a-limited-set of 16 projections. Figure $1 \mathrm{~b}$ shows the same reconstructed slice from a misaligned system. Figures $1 \mathrm{c}$ and $1 \mathrm{~d}$ illustrate the fully reconstructed slices from the aligned and misaligned systems, respectively. Misalignments result in significant artifacts of the reconstructed disc edge. 


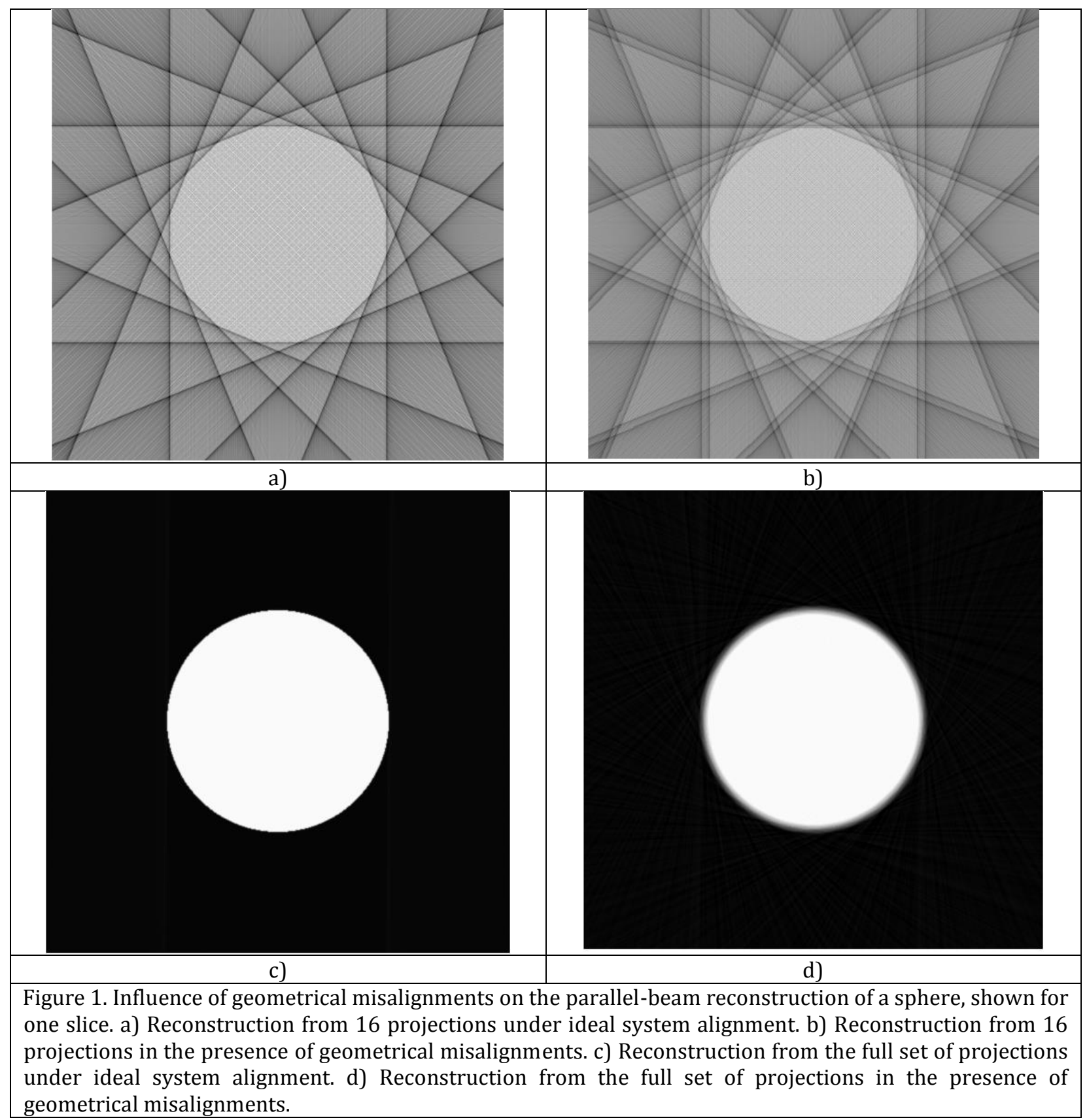

\subsection{Cone-beam geometry model}

The geometrical parameterization of a typical cone-beam CT with a circular scanning trajectory is illustrated in figure 2. A global right-handed Cartesian coordinate frame (GCF) is defined by three mutually orthogonal axes $X, Y$, and $Z$ :

- $Y$ axis is parallel to the rotation axis,

- $Z$ axis passes through the cone-beam vertex (focal spot) and perpendicular to the rotation axis, and

- $X$ axis is given by cross product of $Y$ and $Z$, i.e. $X=(Y \times Z)$.

The coordinate frame origin coincides with the X-ray source focal spot $s$. Hence, $s=\left(s_{x}, s_{y}, s_{z}\right)=$ $(0,0,0)$ in the GCF. 


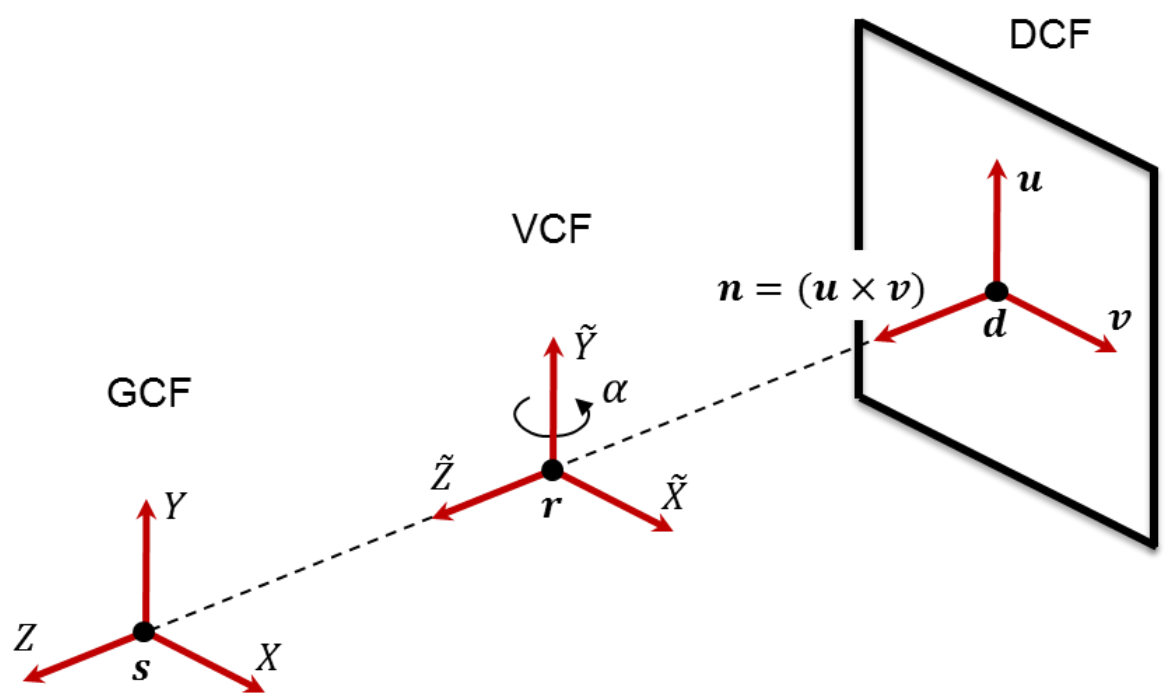

Figure 2. The geometry of a cone-beam CT system is defined by the relative position and orientation of its main components: X-ray source focal spot, axis of object rotation, and detector. Each component is assigned a dedicated coordinate frame. The global coordinate frame (GCF) is fixed on the source focal spot, while the volume coordinate frame (VCF) and detector coordinate frame (DCF) are defined with respect to the GCF.

The detector is assumed to be a rectangular regular planar grid of pixel elements. A detector coordinate frame (DCF) is defined by two orthonormal vectors $\boldsymbol{u}$ and $\boldsymbol{v}$, the origin of which coincides with the geometrical center of the detector $\boldsymbol{d}=\left(x_{d}, y_{d}, z_{d}\right)$. The vectors $\boldsymbol{u}$ and $\boldsymbol{v}$ are parallel to the pixel rows and columns, respectively. The normal to the detector plane $\boldsymbol{n}$ is given by the cross-product of $\boldsymbol{u}$ and $\boldsymbol{v}$, i.e. $\boldsymbol{n}=(\boldsymbol{u} \times \boldsymbol{v})$, and defines the orientation of the detector in the GCF. The set of three vectors $\boldsymbol{u}, \boldsymbol{v}, \boldsymbol{n}$ forms orthonormal basis and can be explicitly parametrized using three extrinsic rotations with respect to GCF in the sequence (1) $\eta$ (rotation about Z axis), (2) $\varphi$ (rotation about $Y$ axis), and (3) $\theta$ (rotation about $X$ axis) [11]. Consequently, detector orientation is given by rotation matrix

$$
\boldsymbol{R}=\left[\begin{array}{l}
(\boldsymbol{u})^{T} \\
(\boldsymbol{v})^{T} \\
(\boldsymbol{n})^{T}
\end{array}\right]=\boldsymbol{R}_{\eta} \boldsymbol{R}_{\varphi} \boldsymbol{R}_{\theta}
$$

where $\boldsymbol{R}_{\eta}, \boldsymbol{R}_{\varphi}$, and $\boldsymbol{R}_{\theta}$ are the elemental rotation matrices that follow.

$$
\boldsymbol{R}_{\eta}=\left[\begin{array}{ccc}
\cos \eta & -\sin \eta & 0 \\
\sin \eta & \cos \eta & 0 \\
0 & 0 & 1
\end{array}\right], \boldsymbol{R}_{\varphi}=\left[\begin{array}{ccc}
\cos \varphi & 0 & -\sin \varphi \\
0 & 1 & 0 \\
\sin \varphi & 0 & \cos \varphi
\end{array}\right], \boldsymbol{R}_{\theta}=\left[\begin{array}{ccc}
1 & 0 & 0 \\
0 & \cos \theta & -\sin \theta \\
0 & \sin \theta & \cos \theta
\end{array}\right]
$$

The orthogonal projection of the source onto the detector $c=\left(u_{c}, v_{c}\right)$ in DCF, typically referred to as the principal point, is given by

$$
\left[\begin{array}{l}
u_{c} \\
v_{c} \\
f
\end{array}\right]=\boldsymbol{R}\left[\begin{array}{llll}
1 & 0 & 0 & -s_{x} \\
0 & 1 & 0 & -s_{y} \\
0 & 0 & 1 & -s_{z}
\end{array}\right]\left[\begin{array}{l}
x_{d} \\
y_{d} \\
z_{d}
\end{array}\right]
$$

where $f$ is the signed orthogonal distance between the source and the detector plane (focal length).

A right-handed Cartesian volume coordinate frame VCF (figure 2) is introduced to address the relative position and orientation of the measurement volume as a function of angular position of the rotation axis. The coordinate axes of the VCF are $\tilde{X}, \tilde{Y}, \tilde{Z}$. During acquisition, the VCF is rotated by a set of angles $\alpha$ distributed from $0^{\circ}$ to $360^{\circ}$ about the $\tilde{Y}$ axis. The direction of positive rotation is given by 
the right-hand screw rule. At the angular position $\alpha=0^{\circ}$, the $\tilde{X}, \tilde{Y}, \tilde{Z}$ axes of the VCF are parallel to the axes of the GCF. The origin of the VCF is given by $r=\left(0,0, z_{r}\right)$ in the GCF.

For a given parameterization, the alignment of a cone-beam CT system with a circular scanning trajectory can be expressed by the following set of conditions.

1. $\boldsymbol{u}=(1,0,0), \boldsymbol{v}=(0,1,0)$ and $\boldsymbol{n}=(0,0,1)$, i.e. $\theta=\varphi=\eta=0^{\circ}$,

2. position of principal point is coincident with detector geometrical center, that is $\boldsymbol{d}=\left(0,0, z_{d}\right)$, and

3. scalars $z_{d}$ and $z_{r}$ are accurately known.

Consequently, geometrical error sources in a typical cone-beam CT system with a circular scanning trajectory can be expressed by a set of seven parameters [10], [11], which are illustrated in figure 3 :

- three detector rotational parameters: $\theta, \varphi, \eta$;

- three detector translational parameters $x_{d}, y_{d}, \varepsilon_{d}$, where $\varepsilon_{d}$ is a detector longitudinal offset of the assumed detector position along $Z$ axis, such that $\boldsymbol{d}=\left(x_{d}, y_{d}, z_{d}+\varepsilon_{d}\right)$;

- longitudinal offset in location of VCF from assumed position $\varepsilon_{r}$, such that $\boldsymbol{r}=\left(0,0, z_{r}+\varepsilon_{r}\right)$.

Positivity of $\varepsilon_{r}$ and $\varepsilon_{d}$ is given by positivity of axes. Positivity of rotations $\theta, \varphi$ and $\eta$ is defined by right-hand screw rule.

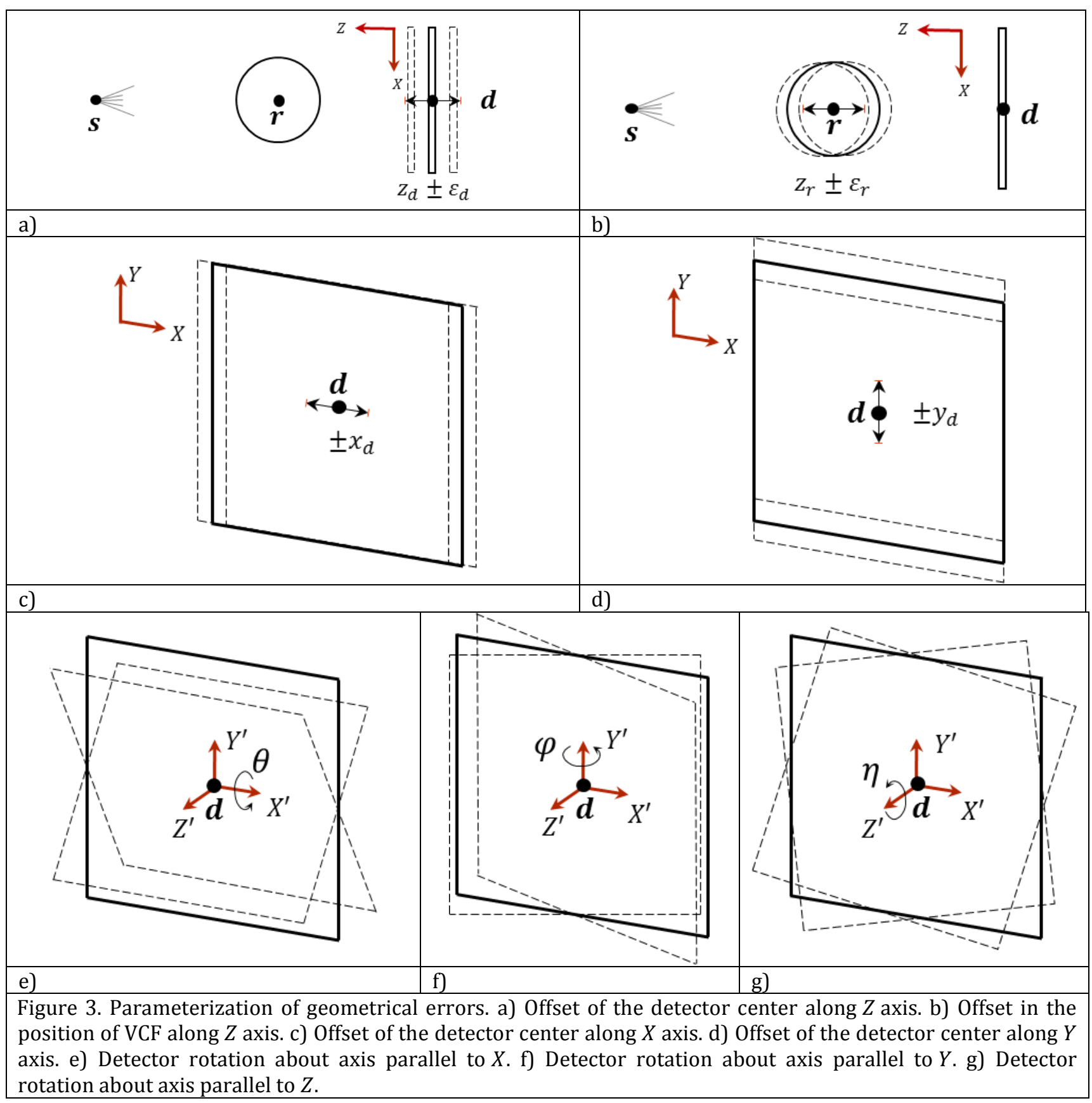




\section{CT geometrical error model}

\subsection{Projection of object edges}

In metrology the dimensional extent of an object is defined by its surface. Let us start by considering single-material object $f(\boldsymbol{g})$, which is a set of all points $\boldsymbol{g}$ embedded by surface $\mathrm{S}$, such that $f(\boldsymbol{g})=1$ if point $\boldsymbol{g}$ is inside $\mathrm{S}$ and $f(\boldsymbol{g})=0$ otherwise. Conventional CT reconstruction and segmentation is then equivalent to acquiring an estimation $S^{*}$ of $S$ in the form of a tomographically reconstructed voxel model. Here and further we assume that the surface of interest is convex, an assumption that can be applied to most dimensional measurement tasks. Note that dimensional measurements of internal cavities such as a cylindrical hole or a half-sphere mold are performed by fitting a cylinder and a sphere, respectively, to the acquired surface points. Therefore, in the context of dimensional metrology, internal features can also be described by convex surfaces.

Object boundaries on the projection define the object's apparent contour. Cone-beam tomographic reconstruction of the object surface can be understood as the intersection of visual cones, each defined by back projection of the apparent contour into 3D space towards the source from all rotation positions $\alpha, \alpha \in[0,360]$. We illustrate this concept in figure 4a. For simplicity, the visualization is restricted to one slice and parallel geometry, in which case the cone apex is located at infinity and the visual cones are illustrated as cylinders. The set of visual cylinders from multiple projections construct shape of the object as it is illustrated in figure $4 \mathrm{~b}$. For cone-beam geometry, the apex of the visual cone coincides with source (figure 4c).

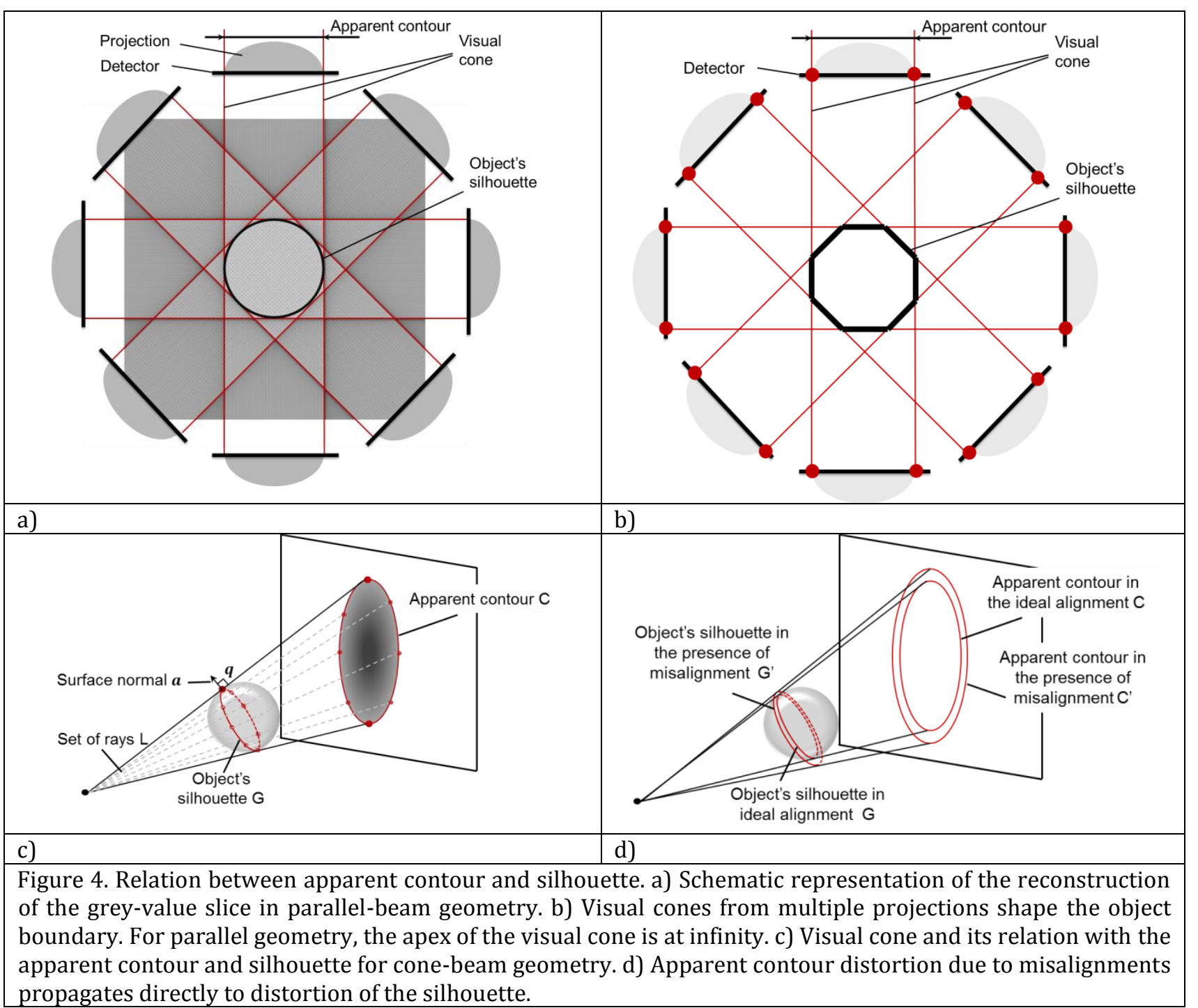


Let us consider surface point $\boldsymbol{q} \in \mathrm{S}$ with surface normal $\boldsymbol{a}$ that has a corresponding contour point $\boldsymbol{c}$ at some viewing perspective. Let us also trace the ray $\boldsymbol{l}$ from source through $\boldsymbol{q}$ towards the detector. The surface normal $\boldsymbol{a}$ will be perpendicular to the ray $\boldsymbol{l}$ and, following the terminology typically used in computer-aided design (CAD), will be called a silhouette point. The relationship between apparent contour and silhouette is illustrated in figure 4c. For a given viewing perspective, the set of all rays $\mathrm{L}$ that are perpendicular to the object's surface will give a subset $\mathrm{G}$ of surface points (points of tangency), which is called object's silhouette.

If the alignment of a CT system satisfies the conditions outlined in section 4.2, for a given rotation position $\alpha$ the apparent contour $\mathrm{C}_{\alpha}$ is back projected correctly to the corresponding silhouette $\mathrm{G}_{\alpha}$ of the object. Let us assume that the CT system is misaligned, i.e. one or more error parameters $\varepsilon_{r}, \varepsilon_{d}, x_{d}, y_{d}, \theta, \varphi, \eta$ (section 4.2) are non-zero, then there will be a discrepancy in the detector coordinates assigned to the apparent contour $\mathrm{C}_{\alpha}{ }_{\alpha}$ when compared to the same coordinates in the aligned system $\mathrm{C}_{\alpha}$. As a result, there will be discrepancy between the real surface $\mathrm{S}$ and the back projected edge $\mathrm{G}_{\alpha}$ (figure $4 \mathrm{~d}$ ). The collection of erroneously back projected edges from all rotation positions of the object $\mathrm{G}_{\alpha}, \forall \alpha \in\left[0^{\circ}, 360^{\circ}\right]$ generates an erroneous surface in the volumetric model of the object, shown as double borders in figure 1. Moreover, there will be discrepancies between apparent contours from different rotation positions; therefore, there will be no object with surface $S$ which can produce the given apparent contours.

\subsection{Geometrical error model}

In this section we introduce a geometrical error model that utilizes the relationship between the apparent contour and the silhouette in the presence of geometrical misalignments to estimate the error in the tomographically reconstructed object surface. The model consists of first forward projecting the silhouette surface point to estimate the location of corresponding apparent contour point on the detector plane in the presence of geometrical misalignment, which is described by seven parameters $\varepsilon_{r}, \varepsilon_{d}, x_{d}, y_{d}, \theta, \varphi, \eta$ (see section 4.2). The second step involves back projecting the estimated apparent contour point with assumption of nominal geometry of the CT system. Forwardand back-projection are repeated for all surface points of the object to be scanned.

\subsubsection{Relationship between silhouette point and rotation position}

In order to define the relationship between silhouette and apparent contour, we first introduce the relationship between silhouette point and rotation position. Let us consider a single surface point $\boldsymbol{q}=\left(q_{x}, q_{y}, q_{z}\right), \boldsymbol{q} \in \mathrm{S}$ in the VCF with surface normal $\boldsymbol{a}=\left(a_{x}, a_{y}, a_{z}\right)$. In the general case, one of two scenarios is possible:

1. There will be no rays from source $\boldsymbol{s}=\left(s_{x}, s_{y}, s_{z}\right)$ that are tangent to $S$ at $\boldsymbol{q}, q_{y} \neq 0$ at any rotation position $\alpha$ for current position of source and detector since cone-beam geometry with circular trajectory does not fulfill the condition for data sufficiency [13], [14]. This scenario occurs if the following inequality is satisfied:

$$
\left(s_{x} a_{z}-s_{z} a_{x}+z_{r} a_{x}\right)^{2}+\left(s_{x} a_{x}+s_{z} a_{z}-z_{r} a_{z}\right)^{2}<\left(q_{x} a_{x}+a_{y}\left(q_{y}-s_{y}\right)+q_{z} a_{z}\right)^{2} .
$$

Consequently, the surface point $\boldsymbol{q}$ will not correspond to a silhouette for any rotation position $\alpha$.

2. There will be two rays tangent to $S$ at $\boldsymbol{q}$ at the rotation positions $\alpha_{1}$ and $\alpha_{2}, \alpha_{1}, \alpha_{2} \leq 360^{\circ}$. In the case of parallel beam scanning geometry, $\alpha_{2}=\alpha_{1}+180^{\circ}$, whereas for cone-beam scanning geometry the relationship between the two rotation positions depends on the position of $\boldsymbol{q}$ in the measurement volume and is given by equation 6 below. 


$$
\begin{aligned}
\alpha_{1,2}= \pm \cos ^{-1} & \left(\frac{q_{x} a_{x}+a_{y}\left(q_{y}-s_{y}\right)+q_{z} a_{z}}{\sqrt{\left(s_{x} a_{z}-s_{z} a_{x}+z_{r} a_{x}\right)^{2}+\left(s_{x} a_{x}+s_{z} a_{z}-z_{r} a_{z}\right)^{2}}}\right) \\
& +\tan ^{-1}\left(\frac{s_{x} a_{x}+s_{z} a_{z}-z_{r} a_{z}}{s_{x} a_{z}-s_{z} a_{x}+z_{r} a_{x}}\right)
\end{aligned}
$$

More information on equations (4) and (5) can be found in [14].

\subsubsection{Forward projection}

Forward projection is the mapping of a three-dimensional volume into a two-dimensional image, in the case of CT the radiograph [15]. Let surface point $\boldsymbol{q}_{(V C F)}=\left(q_{x}, q_{y}, q_{z}\right)_{(V C F)}, \boldsymbol{q}_{(V C F)} \in \mathrm{S}$ with surface normal $\boldsymbol{a}=\left(a_{x}, a_{y}, a_{z}\right)$ satisfy the second condition from section 5.2 .1 (note that parameter $z_{r}$ must be updated if $\varepsilon_{r} \neq 0$ ). To distinguish point coordinates in different coordinate frames, here and further we use corresponding subscripts. According to previous section, there are two rotation positions $\alpha_{1}$ and $\alpha_{2}$ (eq. (5)) in which point $\boldsymbol{q}_{(V C F)}$ becomes a silhouette point $\boldsymbol{q}_{(G C F)}^{\left(\alpha_{1}\right)}$ and $\boldsymbol{q}_{(G C F)}^{\left(\alpha_{2}\right)}$ respectively, as shown in figure 5. For brevity, the equations are shown for the generic rotation position $\alpha$, which is substituted by $\alpha_{1}$ and $\alpha_{2}$ for $\boldsymbol{q}_{(G C F)}^{\left(\alpha_{1}\right)}$ and $\boldsymbol{q}_{(G C F)}^{\left(\alpha_{2}\right)}$, respectively. Given the rotation direction about the $\tilde{Y}$ axis, the location of the point $\boldsymbol{q}_{(G C F)}^{(\alpha)}$ in the GCF is given by

$$
\boldsymbol{q}_{(G C F)}^{(\alpha)}=\left[\begin{array}{c}
q_{x}^{(\alpha)} \\
q_{y}^{(\alpha)} \\
q_{z}^{(\alpha)} \\
1
\end{array}\right]_{(G C F)}=\left[\begin{array}{cccc}
1 & 0 & 0 & 0 \\
0 & 1 & 0 & 0 \\
0 & 0 & 1 & \left(-z_{r}+\varepsilon_{r}\right) \\
0 & 0 & 0 & 1
\end{array}\right]\left[\begin{array}{cccc}
\cos \alpha & 0 & -\sin \alpha & 0 \\
0 & 1 & 0 & 0 \\
\sin \alpha & 0 & \cos \alpha & 0 \\
0 & 0 & 0 & 1
\end{array}\right]\left[\begin{array}{c}
q_{x} \\
q_{y} \\
q_{z} \\
1
\end{array}\right]_{(V C F)} .
$$

Forward projection of point $\boldsymbol{q}_{(G C F)}^{(\alpha)}$ on the detector plane $\boldsymbol{w}_{(D C F)}^{(\alpha)}=\left(w_{u}^{(\alpha)}, w_{v}^{(\alpha)}\right)_{(D C F)}$ can be represented in homogeneous coordinates as

$$
\left[\begin{array}{c}
a w_{u}^{(\alpha)} \\
a w_{v}^{(\alpha)} \\
a
\end{array}\right]=\boldsymbol{P}\left[\begin{array}{c}
q_{x}^{(\alpha)} \\
q_{y}^{(\alpha)} \\
q_{z}^{(\alpha)} \\
1
\end{array}\right]_{(G C F)}=\left[\begin{array}{ccc}
f & 0 & u_{c} \\
0 & f & v_{c} \\
0 & 0 & 1
\end{array}\right]\left[\begin{array}{c}
\left(\boldsymbol{u}^{\prime}\right)^{T} \\
\left(\boldsymbol{v}^{\prime}\right)^{T} \\
\left(\boldsymbol{n}^{\prime}\right)^{T}
\end{array}\right]\left[\begin{array}{cccc}
1 & 0 & 0 & -s_{x} \\
0 & 1 & 0 & -s_{y} \\
0 & 0 & 1 & -s_{z}
\end{array}\right]\left[\begin{array}{c}
q_{x}^{(\alpha)} \\
q_{y}^{(\alpha)} \\
q_{z}^{(\alpha)} \\
1
\end{array}\right]_{(G C F)},
$$

where the position of principal point $c=\left(u_{c}, v_{c}\right)$ and focal length $f$ are given by equation (3) with $\boldsymbol{d}=\left(x_{d}, y_{d}, z_{d}+\varepsilon_{d}\right) ; \boldsymbol{P}$ is the homogeneous projective matrix and $a$ is a homogeneous scaling factor. Converting equation (7) from homogeneous coordinates to the DCF gives,

$$
\boldsymbol{w}_{(D C F)}^{(\alpha)}=\left[\begin{array}{l}
w_{u}^{(\alpha)} \\
w_{v}^{(\alpha)}
\end{array}\right]_{(D C F)}=\left[\begin{array}{l}
a w_{u}^{(\alpha)} / a \\
a w_{v}^{(\alpha)} / a
\end{array}\right]
$$

In figure 5 we show schematically the forward projection onto a misaligned detector. For simplicity, visualization is restricted to the middle plane. 


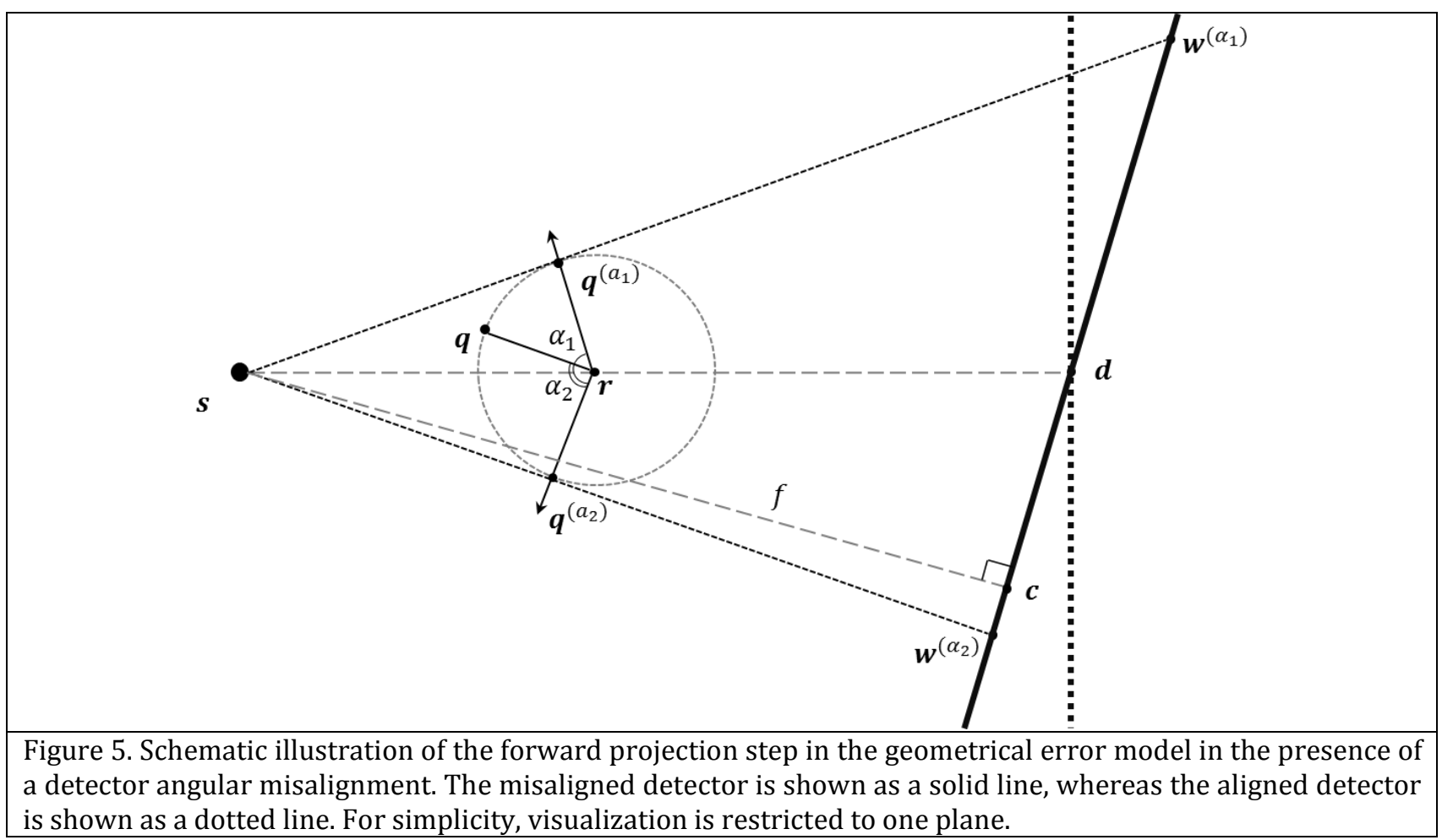

\subsubsection{Back projection}

In figure 6 we show schematic illustration of the back projection step. It was discussed in the section 5.1 that the registered point $\boldsymbol{w}_{(G C F)}^{(\alpha)}$ in the misaligned system has different coordinates $\widehat{\boldsymbol{w}}_{(G C F)}^{(\alpha)}$ in the GCF of aligned system. Coordinates can be related by the following equation:

$$
\widehat{\boldsymbol{w}}_{(G C F)}^{(\alpha)}=\left[\begin{array}{c}
\widehat{w}_{x}^{(\alpha)} \\
\widehat{w}_{y}^{(\alpha)} \\
\widehat{w}_{z}^{(\alpha)}
\end{array}\right]_{(G C F)}=\left[\begin{array}{ccc}
1 & 0 & 0 \\
0 & 1 & 0 \\
0 & 0 & z_{d}
\end{array}\right]\left[\begin{array}{c}
w_{u}^{(\alpha)} \\
w_{v}^{(\alpha)} \\
1
\end{array}\right]_{(D C F)} .
$$

As a result, the set of back projected edge points $\boldsymbol{p}_{(G C F)}^{\left(\alpha_{1}\right)}$ and $\boldsymbol{p}_{(G C F)}^{\left(\alpha_{2}\right)}$ will differ from the actual set of forward projected points $\boldsymbol{q}_{(G C F)}^{\left(\alpha_{1}\right)}$ and $\boldsymbol{q}_{(G C F)}^{\left(\alpha_{2}\right)}$. The homogeneous projective matrix $\boldsymbol{P}$ (equation (7)) is generally not invertible since all the points lying on the ray from source to detector point $\widehat{\boldsymbol{w}}_{(G C F)}^{(\alpha)}$ will be mapped onto $\widehat{\boldsymbol{w}}_{(G C F)}^{(\alpha)}$. Consequently, a new silhouette point $\boldsymbol{p}_{(G C F)}^{(\alpha)}$ will be located along ray $\boldsymbol{l}$ from source $\boldsymbol{s}$ through point $\widehat{\boldsymbol{w}}_{(G C F)}^{(\alpha)}$ (see figure 6). The direction $\boldsymbol{\rho}=\left(\rho_{x}, \rho_{y}, \rho_{z}\right)$ of each ray is then given by

$$
\boldsymbol{\rho}=\frac{\widehat{\boldsymbol{w}}_{(G C F)}^{(\alpha)}-\boldsymbol{s}}{\left\|\widehat{\boldsymbol{w}}_{(G C F)}^{(\alpha)}-\boldsymbol{s}\right\|} .
$$

Deviations in geometrical parameters after effective calibration and compensation are expected to be reasonably small. We therefore assume that local deviations due to the misalignment do not alter the surface profile in the neighborhood of the point $\boldsymbol{q}_{(G C F)}^{(\alpha)}$. The positions of the new silhouette points $\boldsymbol{p}_{(G C F)}^{(\alpha)}$ are then approximated as orthogonal projections of the original silhouette points $\boldsymbol{q}_{(G C F)}^{(\alpha)}$ on vector $\boldsymbol{\rho}$ (equation (11)), i.e. $\boldsymbol{p}_{(G C F)}^{(\alpha)}$ is projected on a line that contains point $\boldsymbol{r}$ and orthogonal to the line from $s$ to $\boldsymbol{w}_{(G C F)}^{(\alpha)}$. 


$$
\boldsymbol{p}_{(G C F)}^{(\alpha)}=\left[\begin{array}{c}
p_{x}^{(\alpha)} \\
p_{y}^{(\alpha)} \\
p_{z}^{(\alpha)}
\end{array}\right]_{(G C F)}=\left\langle-\left(\boldsymbol{q}_{(G C F)}^{(\alpha)}-\boldsymbol{s}\right), \boldsymbol{\rho}\right\rangle\left[\begin{array}{c}
\rho_{x} \\
\rho_{y} \\
\rho_{z}
\end{array}\right]
$$

The location of point $\boldsymbol{p}_{(G C F)}^{(\alpha)}$ in the VCF for rotation position $\alpha=0^{\circ}$ is calculated as

$$
\boldsymbol{p}_{(V C F)}=\left[\begin{array}{l}
p_{x} \\
p_{y} \\
p_{z}
\end{array}\right]_{(V C F)}=\left[\begin{array}{ccc}
\cos \alpha & 0 & \sin \alpha \\
0 & 1 & 0 \\
-\sin \alpha & 0 & \cos \alpha
\end{array}\right]\left[\begin{array}{cccc}
1 & 0 & 0 & 0 \\
0 & 1 & 0 & 0 \\
0 & 0 & 1 & -z_{r}
\end{array}\right]\left[\begin{array}{l}
p_{x}^{(\alpha)} \\
p_{y}^{(\alpha)} \\
p_{z}^{(\alpha)}
\end{array}\right]_{(G C F)}
$$

Substituting $\alpha_{1}$ and $\alpha_{2}$ for the generic rotation position $\alpha$ in equations (9)-(12) gives two new surface points $\boldsymbol{p}_{(V C F)}^{(1)}$ and $\boldsymbol{p}_{(V C F)}^{(2)}$, as shown in figure 6.

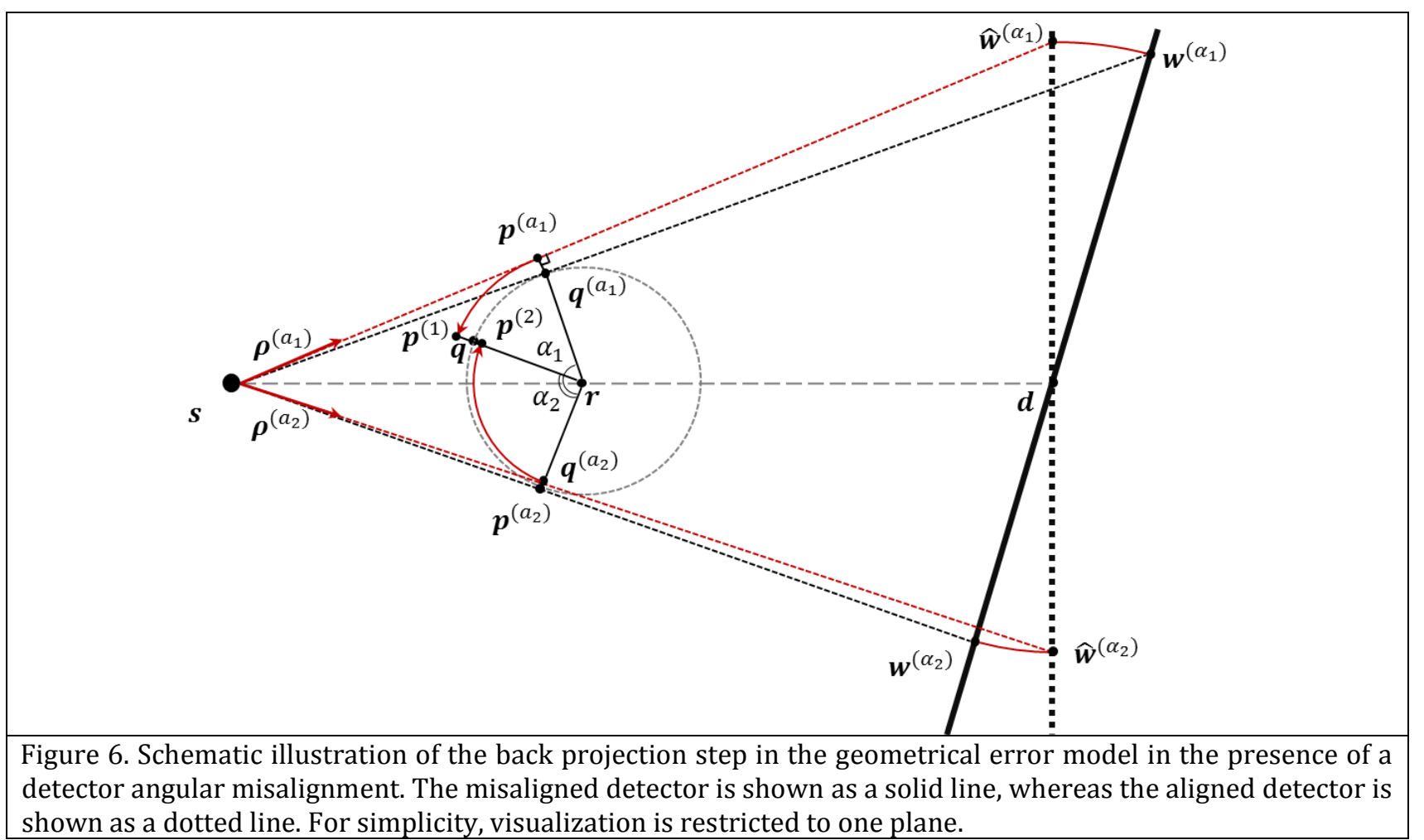

\subsubsection{Application of the geometrical error model for dimensional measurements}

In general, $\boldsymbol{p}^{(1)} \neq \boldsymbol{p}^{(2)} \neq \boldsymbol{q}$ given the discrepancy between radiographs in the presence of misalignments. Consequently, the set of all surface sampling points $S=\{\boldsymbol{q}\}$ will produce two point clouds $\mathrm{P}_{1},=\left\{\boldsymbol{p}^{(1)}\right\}$ and $\mathrm{P}_{2}=\left\{\boldsymbol{p}^{(2)}\right\}$. As was stated in section 5.1, due to the discrepancies between apparent contours from different rotation positions, there will be no single object with surface $S$ which can produce the apparent contours. Hence, in the general case $\mathrm{P}_{1}, \mathrm{P}_{2} \notin \mathrm{S}$, which can result in visually discernible artifacts in the reconstructed volume. In figure $7 \mathrm{a}$, we show the cross-sectional profile of the reconstructed dataset in the presence of severe misalignment superimposed with the rendered point clouds $\mathrm{P}_{1}$ and $\mathrm{P}_{2}$ (solid and dotted lines, respectively) as generated by our geometrical error model (figure $7 \mathrm{~b}$ ). In practice, a CT system with such severe misalignments is unlikely to satisfy the criteria in performance verification (e.g. VDI/VDE 2630 part 1.3 [16]). However, for the purpose of comparing the output of the geometrical error model to simulation results, we utilize such 
misalignments. It should be noted that the geometrical error model does not generate any points on the north and south poles of the sphere since the surface points around the poles do not fulfill the condition for data sufficiency (see section 5.2.1).

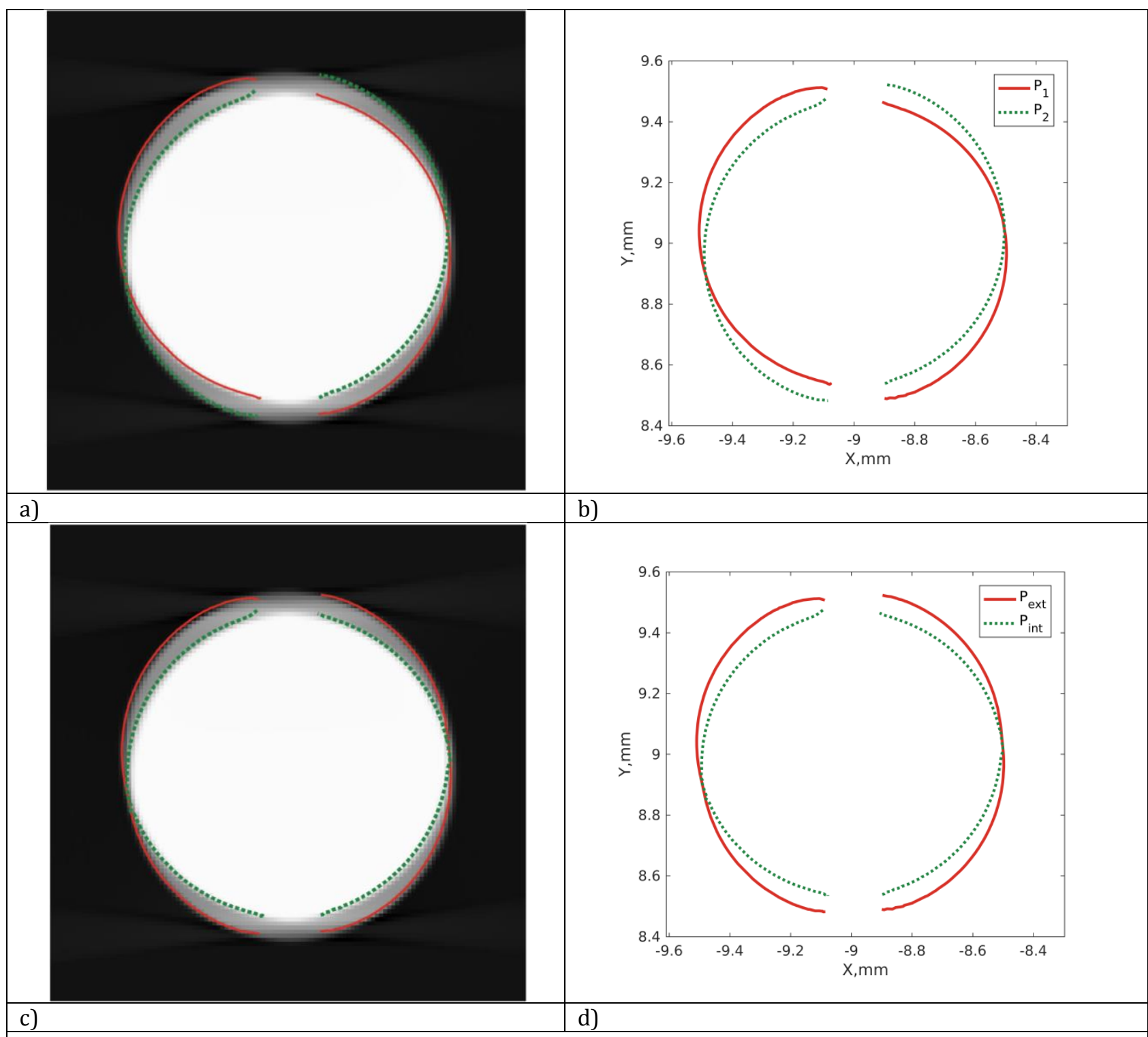

Figure 7. Relationship between simulated and modelled data. a) Grey-value central slice of sphere as output by CT simulation superimposed by output of the geometrical error model. Two overlapping point clouds $P_{1}$ and $P_{2}$ are rendered as solid and dotted lines respectively. b) $\mathrm{P}_{1}$ and $\mathrm{P}_{2}$ are shown separately for better visibility. c) The output of the geometrical error model is categorized in two point clouds $\mathrm{P}_{\text {ext }}$ and $\mathrm{P}_{\text {int }}$, which are shown as solid and dotted outlines, respectively. d) $\mathrm{P}_{\text {ext }}$ and $\mathrm{P}_{\text {int }}$ are shown separately for better visibility.

In figure $7 \mathrm{a}$ the conjunction of volumes enclosed by $\mathrm{P}_{1}$ and $\mathrm{P}_{2}$ corresponds to the volumetric region for which all projections were consistent and hence has relatively higher attenuation value. The exclusive disjunction of volumes enclosed by $\mathrm{P}_{1}$ and $\mathrm{P}_{2}$ gives the volumetric region where projections were not self-consistent thus resulting in "ghost", or relatively lower attenuation, material. The grey value of the "ghost" material corresponds to approximately $50 \%$ of grey value range, i.e. due to the inconsistency of the recorded ray integrals from opposite rotation positions (see section 5.1), backprojected grey values do not complement each other within the reconstruction. Even though $\mathrm{P}_{1}$ and $\mathrm{P}_{2}$ provide valuable information about the influence of the misalignment on the reconstructed volume, they cannot be used for dimensional measurements since neither of them follows volumetric region with homogeneous material. That is, in figure $7 \mathrm{a}$ in the north-east quadrant $\mathrm{P}_{2}$ follows "ghost" material, whereas in the north-west quadrant $\mathrm{P}_{2}$ keeps to high attenuation volumetric region. Therefore, we categorize all points from sets $\mathrm{P}_{1}$ and $\mathrm{P}_{2}$ into an internal point cloud $\mathrm{P}_{\text {int }}$ which envelops 
high attenuation grey value area and corresponds to an object's contour with approximately $75 \%$ grey value gradient, and an external point cloud $\mathrm{P}_{\text {ext }}$ which envelops the lower-attenuation "ghost" material and corresponds to an object's contour with approximately $25 \%$ grey value gradient. In figure $7 \mathrm{c}, \mathrm{d}$, $\mathrm{P}_{\text {ext }}$ and $\mathrm{P}_{\text {int }}$ are shown as solid and dotted outlines, respectively.

If the magnitude of geometrical misalignment is reasonably small, then the gap between $\mathrm{P}_{\mathrm{ext}}$ and $\mathrm{P}_{\text {int }}$ becomes less visually pronounced. In figure $8 \mathrm{a}, \mathrm{b}$, we show the cross-sectional profile of the reconstructed dataset, which is less affected by the misalignment-induced artifacts. Even though misalignment-induced artifacts contribute to an overall perception of a blurred image, this blur cannot be compensated by image deblurring [17] because the point spread function needed for compensation fails to represent the effects of geometrical misalignment.

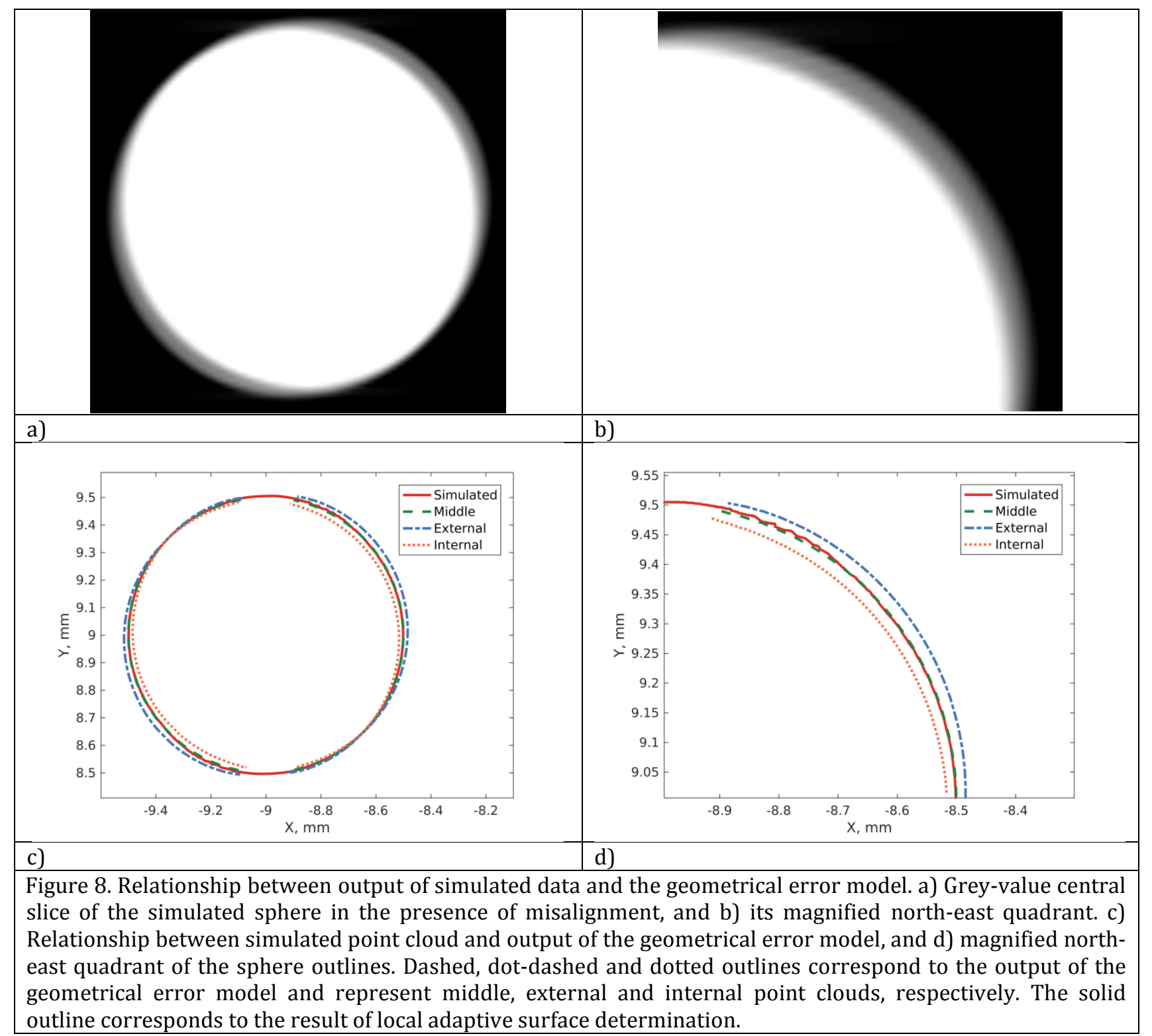

Since $\mathrm{P}_{\text {ext }}$ and $\mathrm{P}_{\text {int }}$ correspond to approximately $25 \%$ and $75 \%$ grey value gradient, respectively, we use them to estimate the location of the surface that would be segmented with the conventional $50 \%$ grey value gradient. We therefore introduce a middle point cloud $\mathrm{P}_{\text {mid }}$, which is located between $\mathrm{P}_{\mathrm{ext}}$ and $\mathrm{P}_{\text {int }}$. In figure 8c,d, we show outlines generated from four point cloud datasets. The dashed, dot-dashed and dotted point cloud outlines correspond to the output of the geometrical error model and represent the middle, external, and internal point clouds, respectively. The solid outline corresponds to the result of local adaptive surface determination applied to the simulated dataset shown in figure 8a,b. A saw-like pattern along the simulated outline (solid line) is observed in 
figure $8 \mathrm{~d}$ and is due to the partial volume effect and discretization error introduced by CT simulation and reconstruction.

The proposed geometrical error model is a computationally efficient alternative to determining the influence of geometrical misalignments on dimensional measurements. Since every surface point is processed separately, the proposed method is highly parallel and is well suited for a fast implementation on modern parallel architectures.

\section{Evaluation of the geometrical error model on simulated data}

The efficacy of the geometrical error model introduced in section 5 was investigated by means of computer simulation. We compare the output of the geometrical error model, i.e. the set of modelled surface coordinate errors, with observed surface coordinate errors in the simulated CT measurement of a dedicated object. Henceforth, we refer to the output of CT simulation as simulated point cloud and to the output of the geometrical error model as modelled point cloud.

In this study, we focus on the application of our model to the CT measurement of a simple object consisting of spheres in a particular arrangement. This initial application serves as a proof of concept, which can later be adapted for measurement of other more complex objects. In order to demonstrate the effectiveness of the method, we compare simulated and modelled point clouds against nominal point cloud in the presence of various geometrical error sources. Dimensional measurements are then performed on both simulated and modelled point clouds.

\subsection{Simulation and evaluation design}

Evaluation object

The evaluation object is shown in figure 9. The evaluation object comprises thirteen spheres of $1 \mathrm{~mm}$ diameter arranged along a diagonal line. At the $\alpha=0^{\circ}$ position, the sphere centers occupy the $Z Y$ plane of the measurement volume. The spheres are numbered 1 to 13 from the top to the bottom (along $Y$ ) of the diagonal. The center of sphere 7 coincides with center of the volume. Spacing between adjacent spheres is $1.5 \mathrm{~mm}$ along the horizontal $(Z)$ direction and $1.5 \mathrm{~mm}$ along the vertical $(Y)$ direction. The diagonal distance between adjacent spheres is therefore approximately $2.121 \mathrm{~mm}$. The proposed geometrical error model is evaluated for various individual geometrical error sources, shown in table 1. 


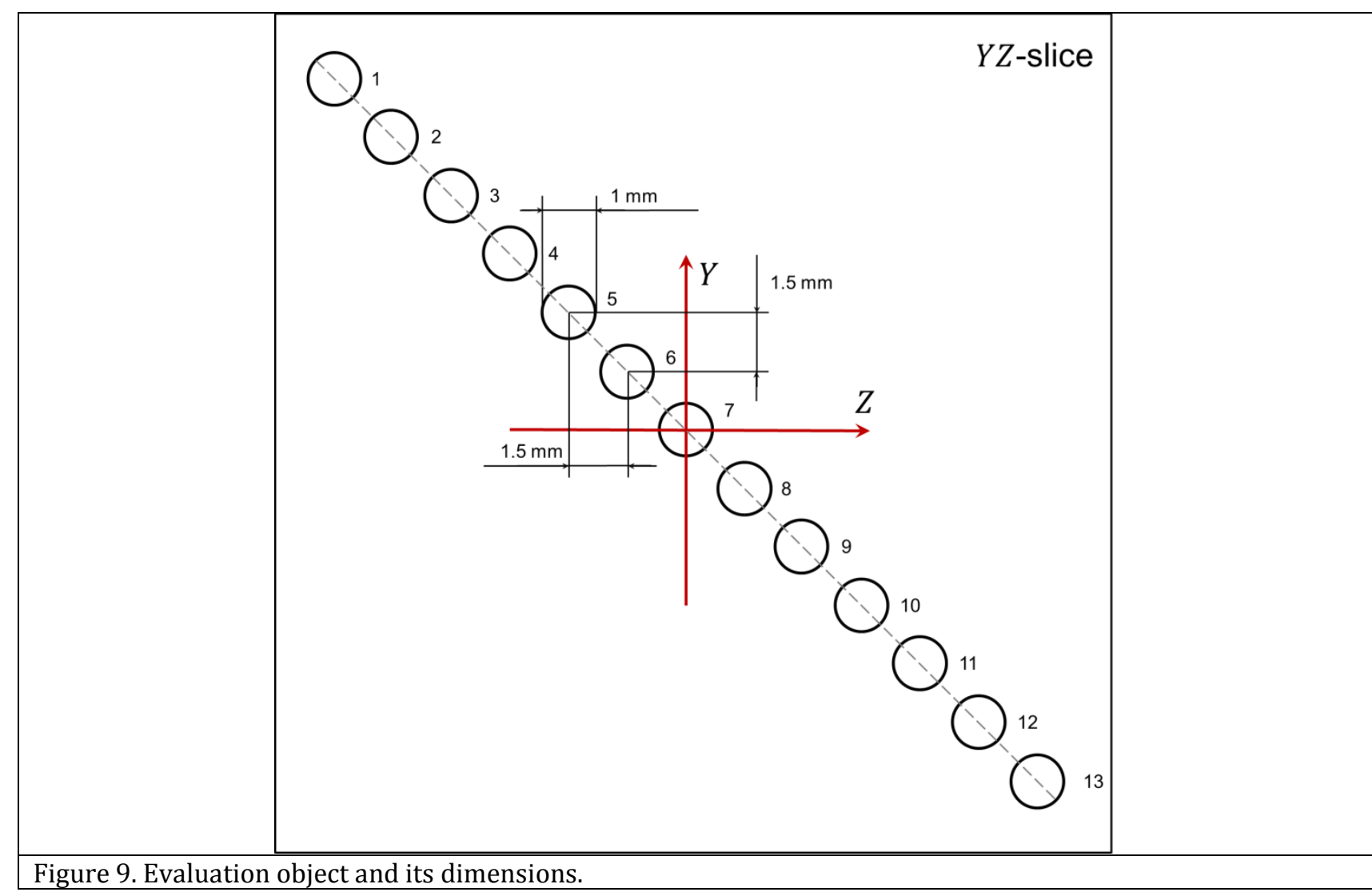

Table 1. Errors in geometrical parameters

\begin{tabular}{|l|l|}
\hline Error source & Value \\
\hline$\theta$ & $1^{\circ}$ \\
\hline$\varphi$ & $0.5^{\circ}$ \\
\hline$\eta$ & $0.1^{\circ}$ \\
\hline$x_{d}$ & $0.25 \mathrm{~mm}$ \\
\hline$y_{d}$ & $0.25 \mathrm{~mm}$ \\
\hline$\varepsilon_{d}$ & $2 \mathrm{~mm}$ \\
\hline$\varepsilon_{r}$ & $0.1 \mathrm{~mm}$ \\
\hline
\end{tabular}

\section{Simulated point cloud}

Computer simulations were carried out for the cone-beam geometry described in section 5.2. A set of radiographs of the reference object was generated in the presence of each error source shown in the table 1, separately. Other acquisition parameters used in the simulation are shown in table 2. Each CT dataset consists of 3600 projections of equally-spaced rotation positions of the reference object, corresponding to an angular increment $\Delta \alpha=0.1^{\circ}$ between consecutive projections. Simulation of the radiographic dataset was performed using an in-house simulation software based on an Intel Embree ray-tracing kernel [18]. Each sphere in the reference object was represented as a triangulated surface. The upper bound on surface normal error was set to $1^{\circ}$, which resulted in approximately $5 \times$ $10^{5}$ triangles per sphere. To eliminate any other error sources, we simulated a monochromatic X-ray spectrum, a point-like focal spot, and noise-free radiographs. For each detector pixel, 16 rays were traced and the average of the calculated intensities was assigned as a single pixel intensity.

Tomographic reconstruction from the simulated radiographs was performed by FDK algorithm with a Ramp filter on Nikon Metrology XT Software Suite. Segmentation of the volumetric data and point cloud sampling was performed in VGStudio MAX (Volume Graphics, GmbH). Local adaptive 
surface determination algorithm with default 4.0 voxels search distance was used for segmentation. The initial contour value was selected as the arithmetic mean of the grey value corresponding to the background and the grey value corresponding to material peak in the grey-value histogram. Surfaces were converted to point clouds in VGStudioMAX using "manual" mode with a volumetric sampling interval approximately equal to half of the voxel size $(6 \mu \mathrm{m})$.

Table 2. Acquisition parameters common to all simulated datasets.

\begin{tabular}{|l|l|}
\hline Parameter & Value \\
\hline Detector size & $400 \times 400 \mathrm{~mm}$ \\
\hline Number of pixels & $2000 \times 2000$ \\
\hline Pixel size & $0.2 \mathrm{~mm}$ \\
\hline Bit-depth & $16 \mathrm{bit}$ \\
\hline$z_{d}$ & $1060 \mathrm{~mm}$ \\
\hline$z_{r}$ & $66.25 \mathrm{~mm}$ \\
\hline Number of voxels & $2000 \times 2000 \times 2000$ \\
\hline Voxel size & $12.5 \mu \mathrm{m}$ \\
\hline Number of projections & 3600 \\
\hline Angular step & $0.1^{\circ}$ \\
\hline
\end{tabular}

\section{Modelled point cloud}

The evaluation object shown in figure 9 was represented as a point cloud with a corresponding surface normal at each data point. Following the approach described in [19], $10^{4}$ random points uniformly distributed on a sphere surface were generated for every sphere. Surface points that satisfy condition (4), section 5.2.1, were not evaluated. The set of modelled point clouds $\mathrm{P}_{\text {ext }}, \mathrm{P}_{\text {int }}$ and $\mathrm{P}_{\text {mid }}$ were calculated according to equations (6)-(12) for every geometrical error source shown in table 1

\subsection{Misalignment-induced artifacts}

Geometrical misalignments affect the reconstructed data differently: some result in visually discernible artifacts in the reconstructed volume, while others do not present visually discernable artifacts, though still affect dimensional measurements. In the presence of $\theta, y_{d}, \varepsilon_{d}$ and $\varepsilon_{r}$, there were no visually discernable double borders observed, i.e. all point cloud outlines $\left(\mathrm{P}_{\text {ext }}, \mathrm{P}_{\text {int }}, \mathrm{P}_{\text {mid }}\right)$ appear to coincide. For this reason, the figures for visual effects due to $\theta, y_{d}, \varepsilon_{d}$ and $\varepsilon_{r}$ are not presented. In the next sections it will be shown that, while these error sources have relatively negligible influence on the reconstructed sphere form, their influence on reconstructed sphere size and sphere position within the measurement volume is significant.

In the presence of $\varphi, \eta$ and $x_{d}$, non-symmetrical double-borders are observed as shown in figure 10 left column. In figure 10, right column, the color of sphere surface points is rendered based on the orthogonal distance between $\mathrm{P}_{\text {int }}$ and $\mathrm{P}_{\text {ext }}$. The double-borders effect is not uniform along the sphere's edge and its appearance strongly depends on the type of error source. The visual comparison of greyvalue slices to rendered point clouds in figure 10 demonstrates that the geometrical error model captures the systematic behavior of the corresponding misalignment. 


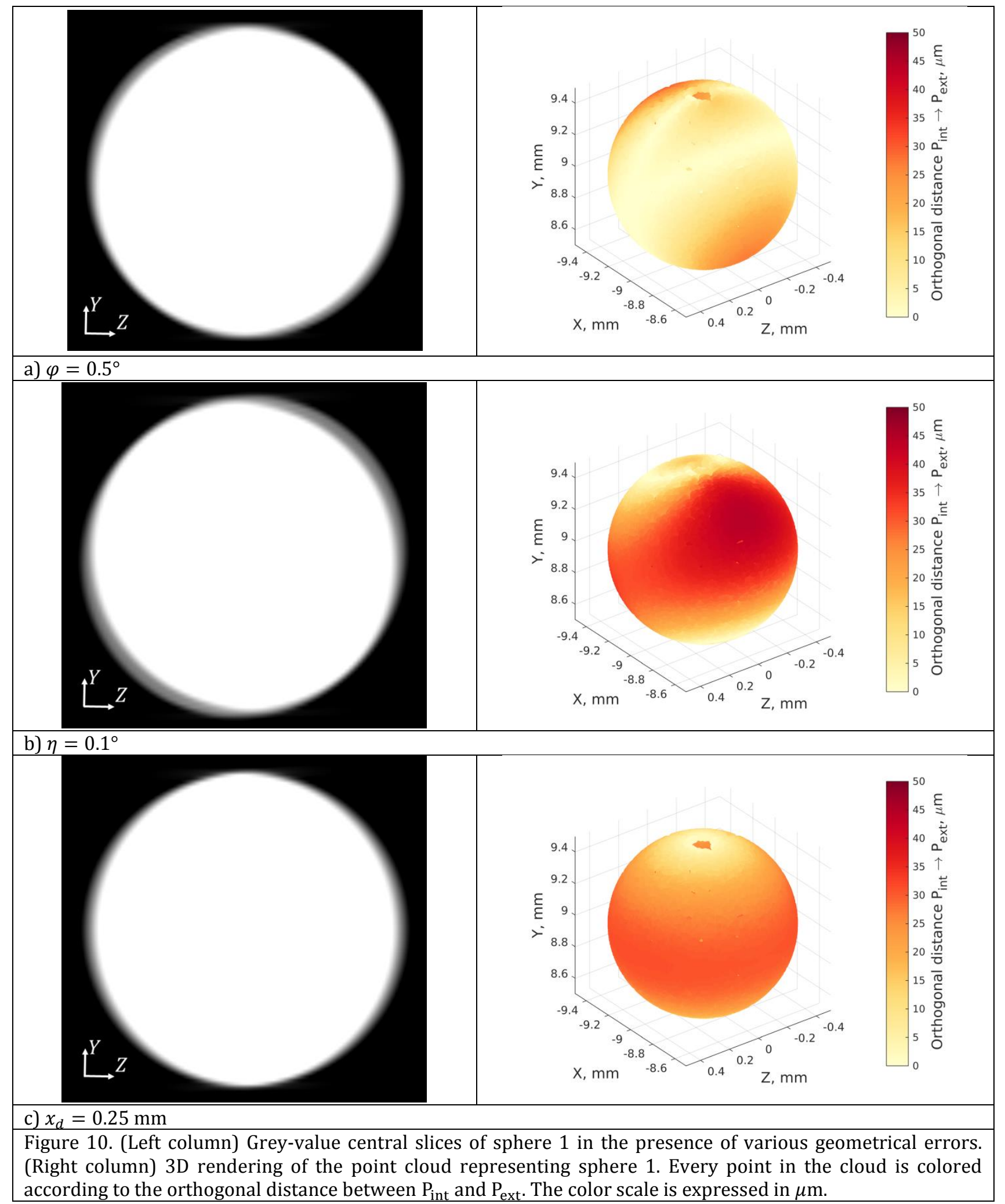

\subsection{Quantitative comparison of modelled and simulated point cloud data}

To investigate the agreement between modelled and simulated point clouds, a comparison was made of the middle point cloud $\mathrm{P}_{\text {mid }}$ (section 5.2.4) and the point cloud obtained from VG Studio MAX against a nominal point cloud representing the evaluation object. The nominal point cloud was obtained by random sampling of an ideal sphere surface (section 6.1) at the reference positions of the spheres (figure 9), therefore the nominal point cloud is considered to be free from errors. All three point clouds contained $N_{p}=\sim 10^{4}$ points per sphere. 
To enable this comparison, we implemented a point cloud comparison procedure in MATLAB (The MathWorks, USA). For every point $\boldsymbol{p}_{i}=\left(p_{x}, p_{y}, p_{z}\right), i=1, \ldots, N_{p}$ in the nominal point cloud, the 200 nearest points $\boldsymbol{q}_{j}=\left(q_{x}, q_{y}, q_{z}\right), j=1, \ldots, 200$, were found in the corresponding point cloud using $k n n$-search [20]. Then, a quadratic surface in the form

$$
c_{0} q_{x}^{2}+c_{1} q_{y}^{2}+c_{2} q_{z}^{2}+c_{3} q_{x} q_{y}+c_{4} q_{y} q_{z}+c_{5} q_{x} q_{z}+c_{6} q_{x}+c_{7} q_{y}+c_{8} q_{z}+c_{9}=0
$$

was fitted to points $\boldsymbol{q}_{j}$ using the Taubin method [21]. The signed distance $d_{i}, i=1, \ldots, N_{p}$ between $\boldsymbol{p}_{i}$ and the fitted surface was calculated for every point $\boldsymbol{p}_{i}$ [22]. Positive deviations correspond to distance vectors that point outward from the surface of the nominal point cloud.

Figure 11, left column, shows histograms of signed distances $d_{i}$ between the nominal and simulated point clouds for the sphere 1 in the absence of any geometrical errors (figure 11a) and in the presence of each error source presented in table 1 (figure 11b-h), whereas the right column shows comparison between modelled and nominal point clouds under the same scenarios. The mean value and corresponding 95\% confidence interval (based on $2.5 \%$ and $97.5 \%$ quantile) are shown as solid and dashed lines, respectively.

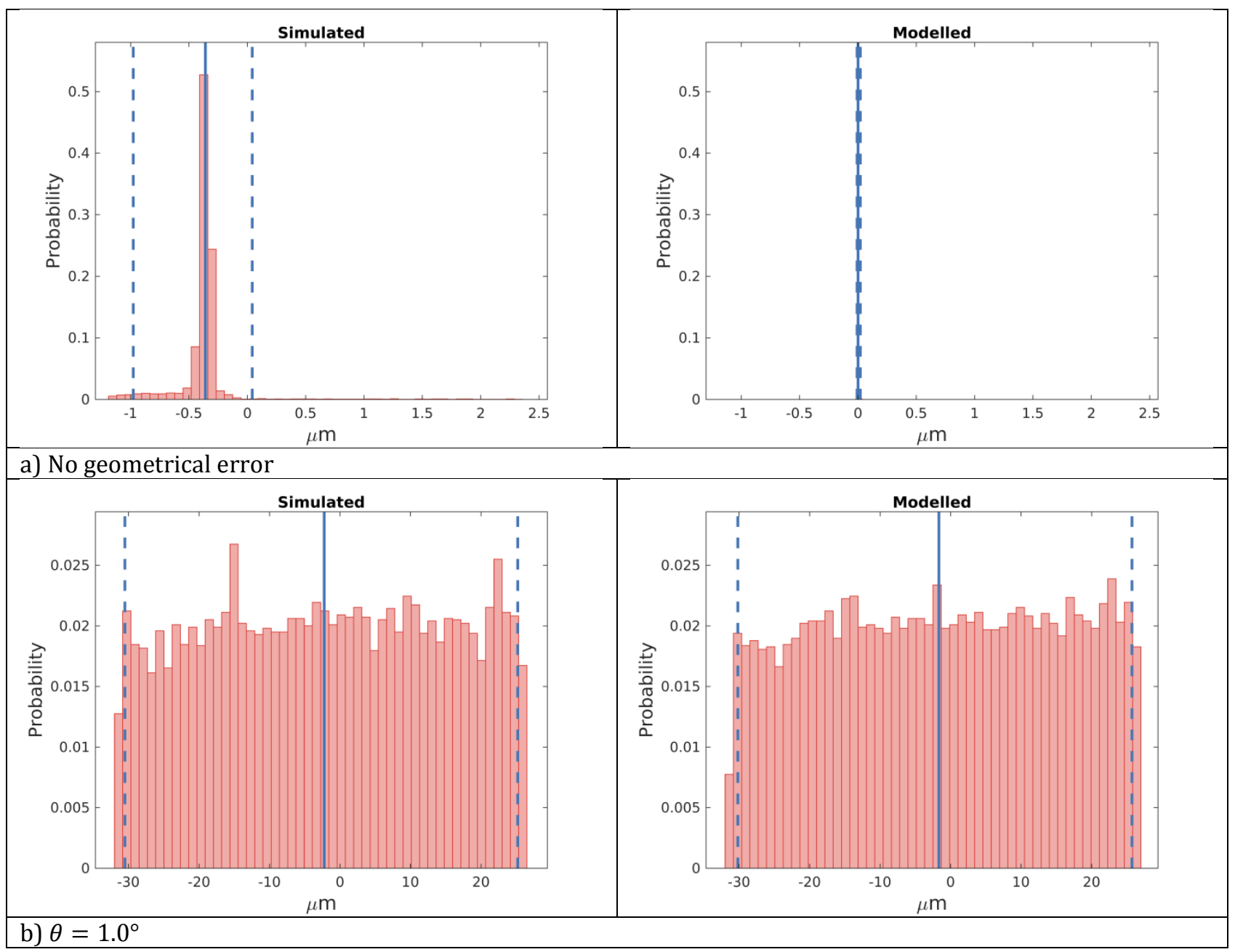




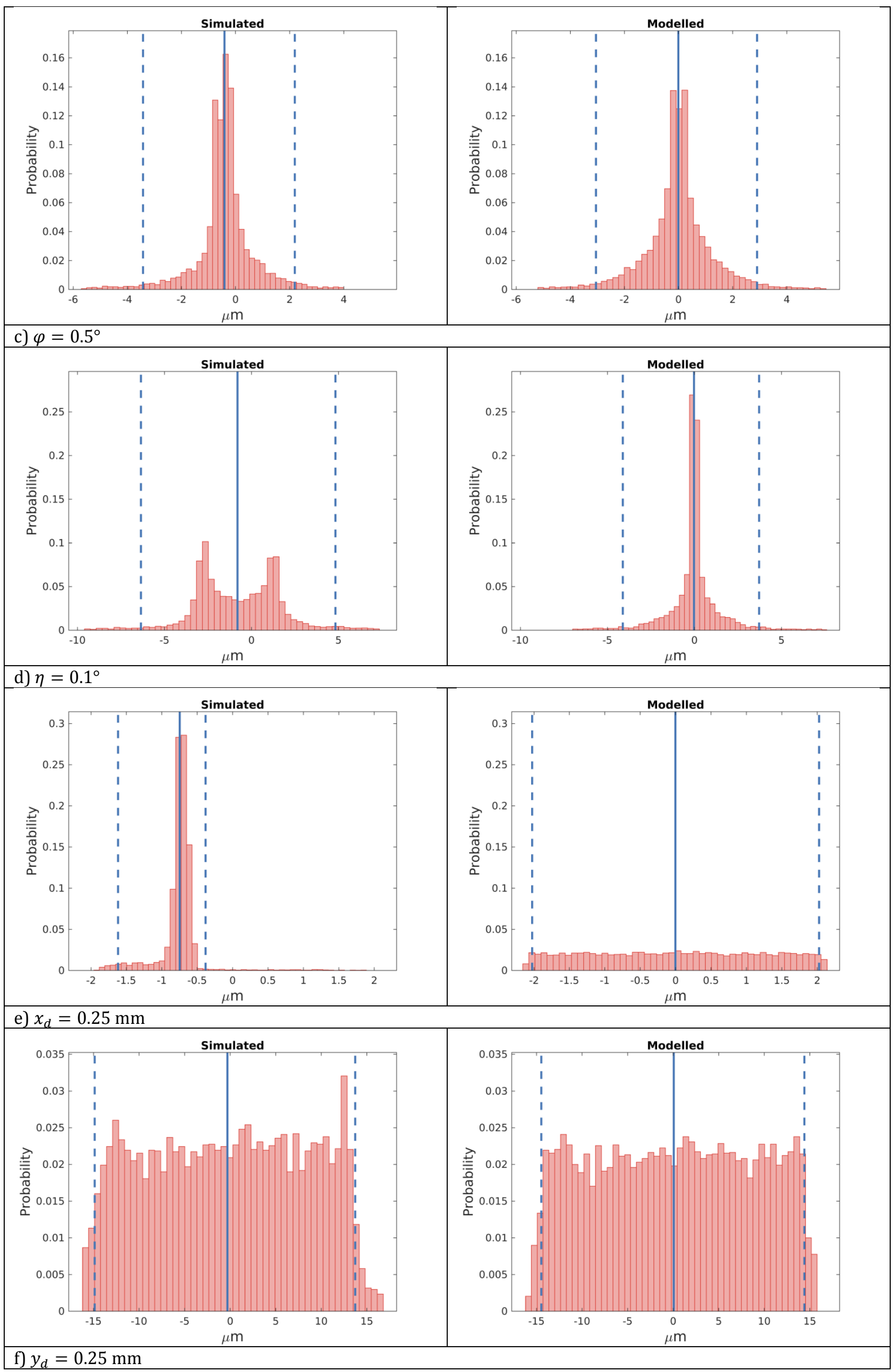



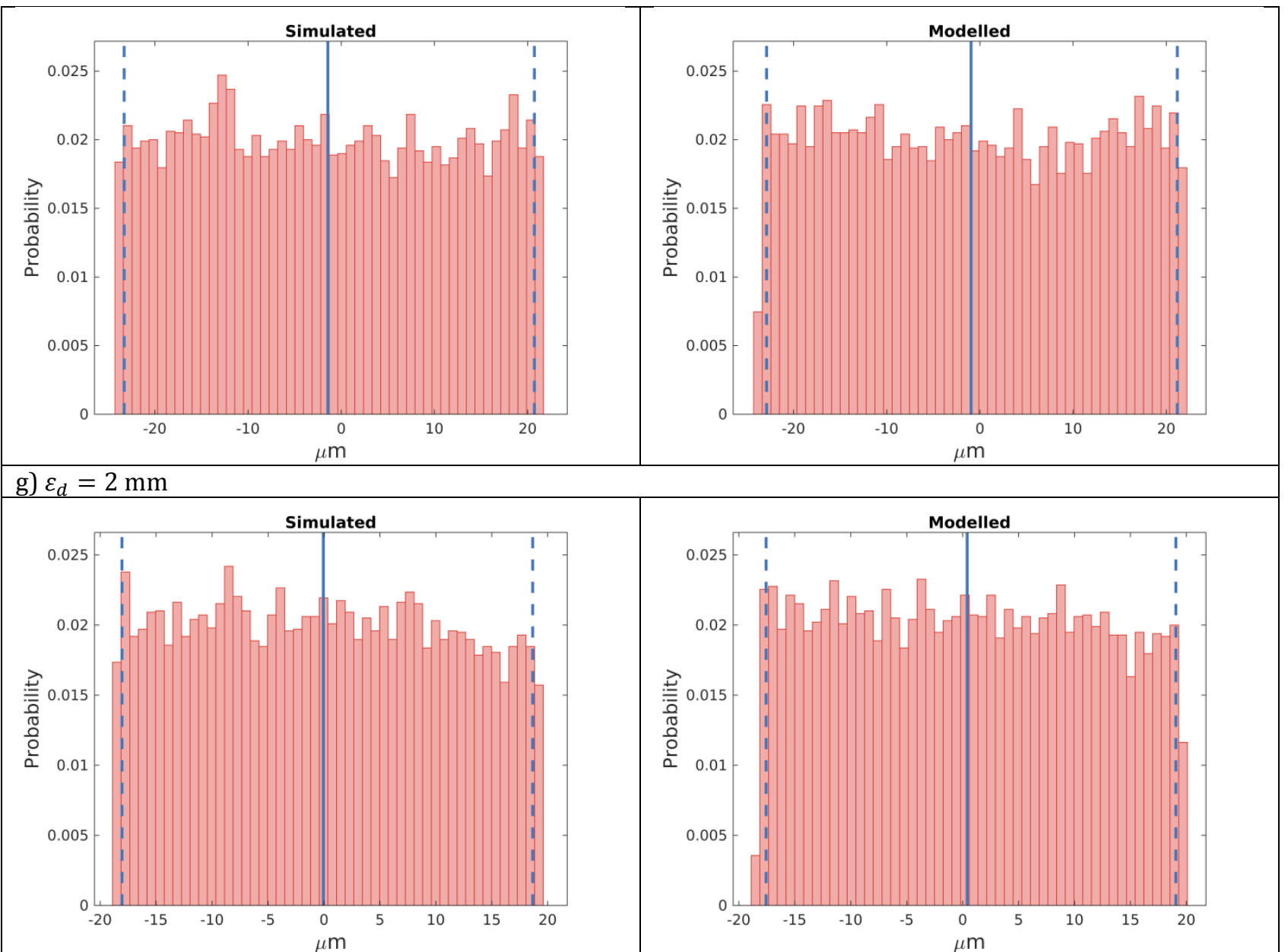

h) $\varepsilon_{r}=0.1 \mathrm{~mm}$

Figure 11. Histogram of signed distances between simulated and nominal point clouds (left column) and between modelled and nominal point clouds (right column) for sphere 1. Mean values and corresponding 95\% confidence interval (based on $2.5 \%$ and $97.5 \%$ quantile) are shown as solid and dashed lines, respectively. Histograms are normalized by probability, i.e. the sum of the bar heights is less than or equal to 1.

The results in the absence of any misalignment for the simulated point cloud (figure 11a, left) indicate that simulation accurately recovers the surface of the evaluation object and can therefore be used as the reference for comparison. The statistical dispersion of signed distances for the modelled point cloud (figure 11a, right) shows that rounding errors in the computational steps of the geometrical error model and the point cloud comparison method do not introduce significant errors.

Results for $\theta$ (figure 11b), $\varphi$ (figure 11c), $y_{d}$ (figure 11f), $\varepsilon_{d}$ (figure 11g), and $\varepsilon_{r}$ (figure 11f) show excellent agreement between simulated and modelled point clouds in terms of both distribution shape and dispersion. For $\eta$ (figure 11d) and $x_{d}$ (figure11 g), however, there is slight disagreement in results. Distribution of signed distances for $\eta$ in the simulated point cloud (figure 11d, left) has a bimodal shape, whereas signed distances in modelled point cloud obey a unimodal distribution (figure 11d, right). This observed behavior may originate from dependence of the effects of $\eta$ and the partial volume effect. Despite the fact that $x_{d}$ introduces significant double-borders, as was shown in figure 10c, the dispersion of signed distances in simulated data is lower than in modelled data (figure $11 \mathrm{~g}$ ). This disagreement is likely due to the fact that the influence of $x_{d}$ is partially compensated by the local adaptive surface determination applied to the simulated data.

In appendix A, figure A.1, we show the rendering of point clouds corresponding to sphere 1 colored according to the magnitude of signed distances $d_{i}$ in the presence of the error sources listed in table 1. Rendered point clouds indicate that the geometrical error model captures the principal influence of geometrical error sources on the reconstructed object's surface. A summary of statistical information for all spheres 1-13 is provided as error-bar plots in appendix A, figure A.2. The results show that the output of the geometrical error model agrees with the CT simulation output. 


\subsection{Comparison in terms of dimensional measurements}

We define quantitative criteria for determining dimensional accuracy of the reconstructed volume. These criteria are center-to-center distances between sphere 1 and consecutive spheres and individual sphere roundness errors. Center position of sphere is assessed using least squares fit; radii of inscribed and circumscribed concentric spheres are determined by the minimum-zone method [23]. In figure 12, we show absolute error in center-to-center distances between sphere 1 and all consecutive spheres 2-13, i.e. point 5 in the plot corresponds to error in the distance between spheres 1 and 5 . Modelled data is shown as filled circles, whereas simulated data is shown as crosses. Figure 12 indicates that the difference between distances measured on modelled and simulated data is negligible.

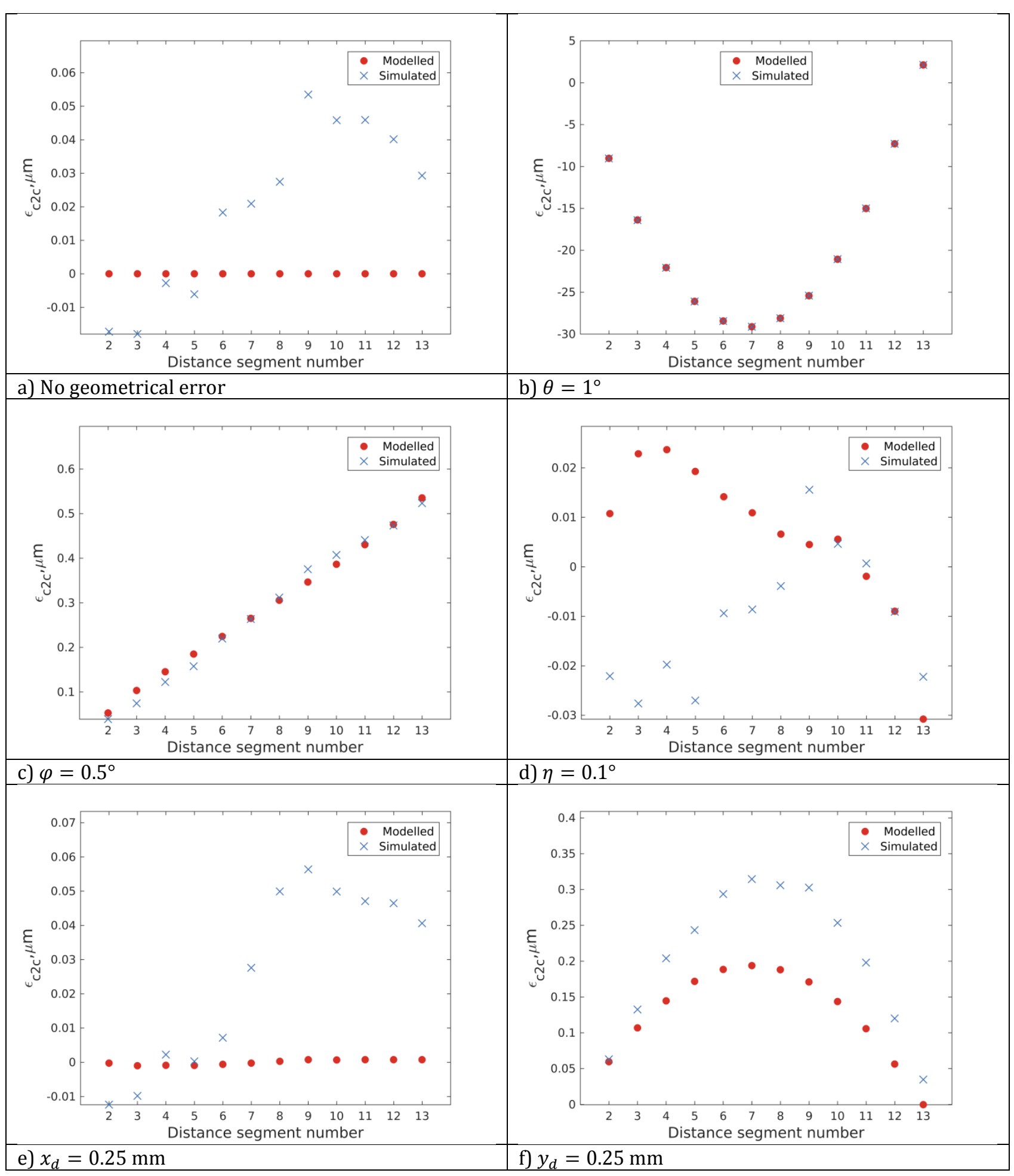


1

2

3

4

5

6

7

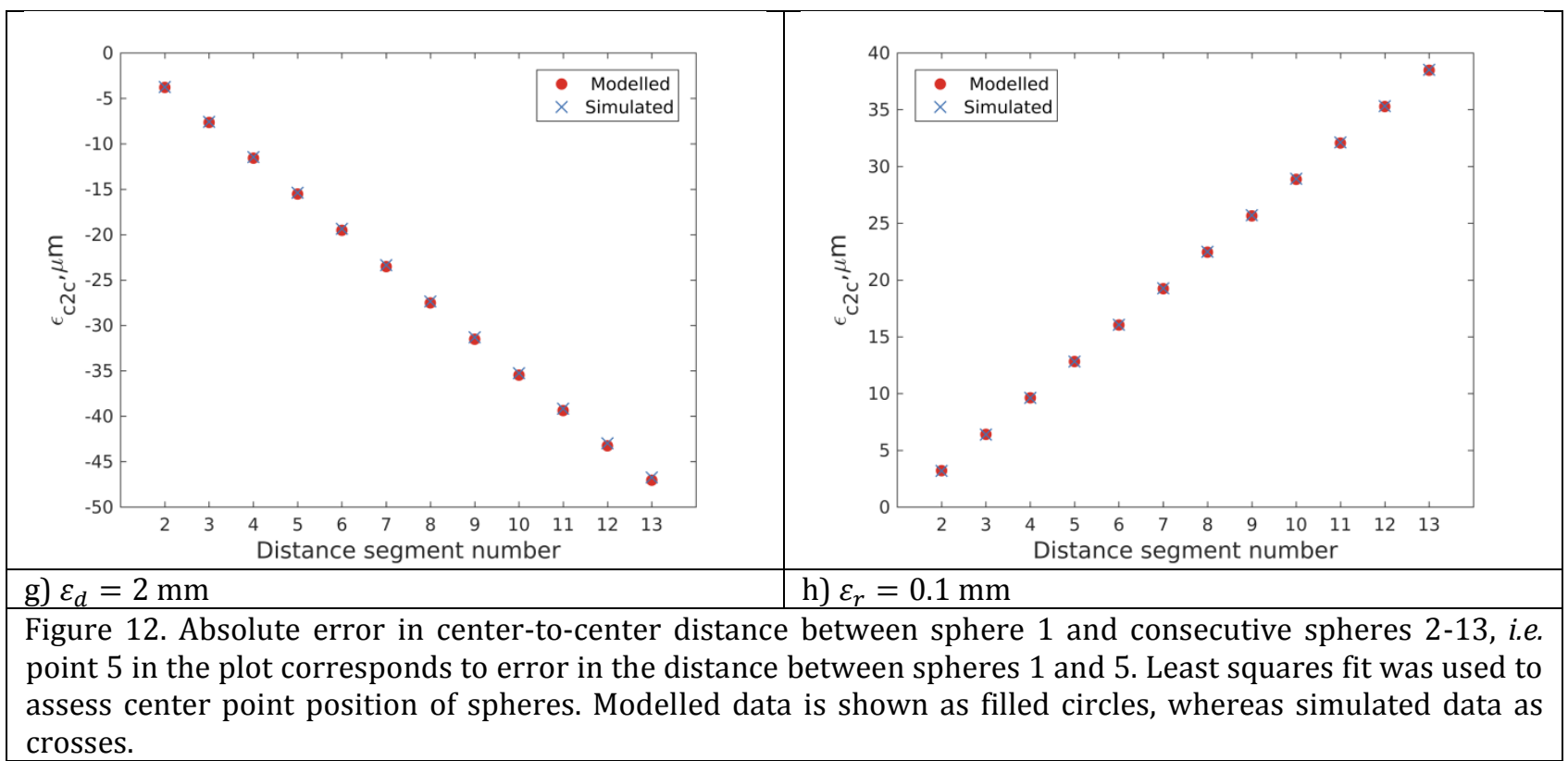

Figure 13 presents the inscribed and circumscribed radii. Modelled data is shown as filled circles and squares; simulated data is depicted as crosses and plus signs. The dashed line corresponds to the reference radius. In the absence of any misalignment (figure 13a) roundness error increases with increasing distance of the reconstructed sphere from the midplane, i.e. the $X Z$-plane containing the X-ray source. This behavior is due to the degradation of tomographically reconstructed edges on the south and north poles of spheres caused by cone-beam artifacts [13]. The effects of cone-beam artifacts are noticeable also in the simulated results in the presence of geometrical misalignments (figure 13b-h). The geometrical error model catches both deviation in radii and sphere roundness errors up to aforementioned behavior due to cone-beam artifacts.

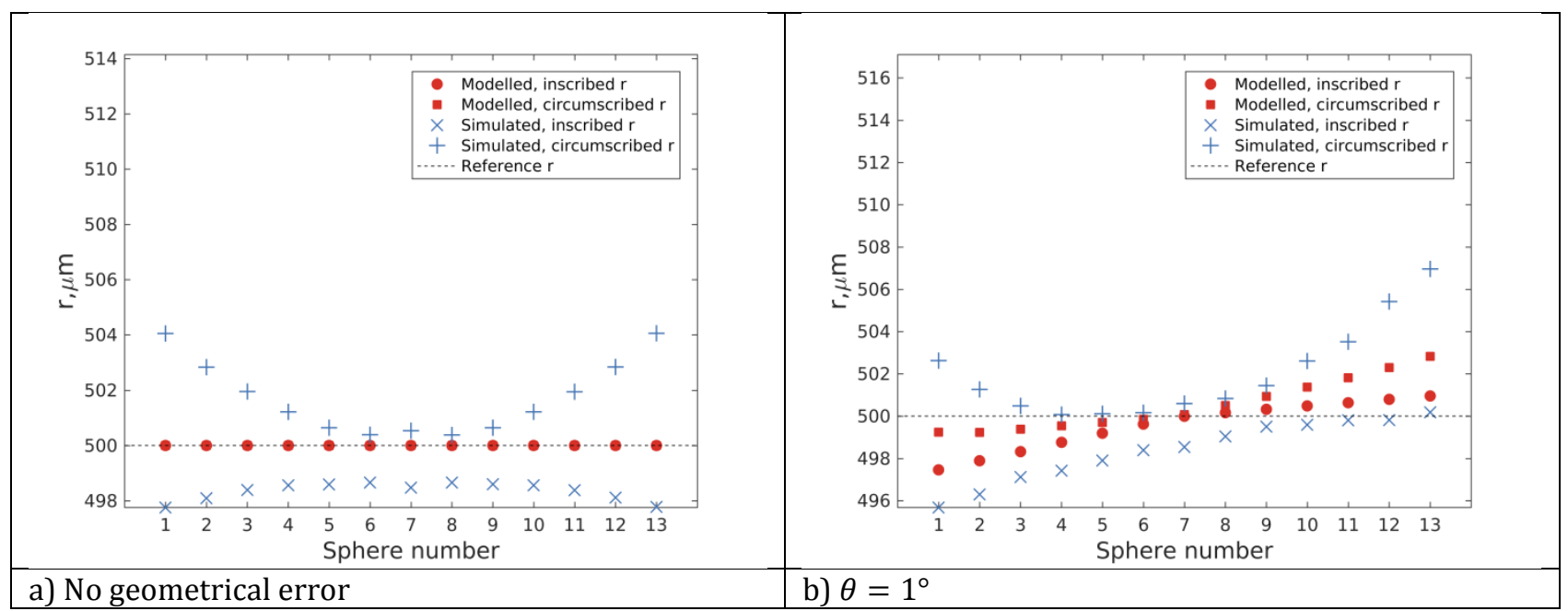




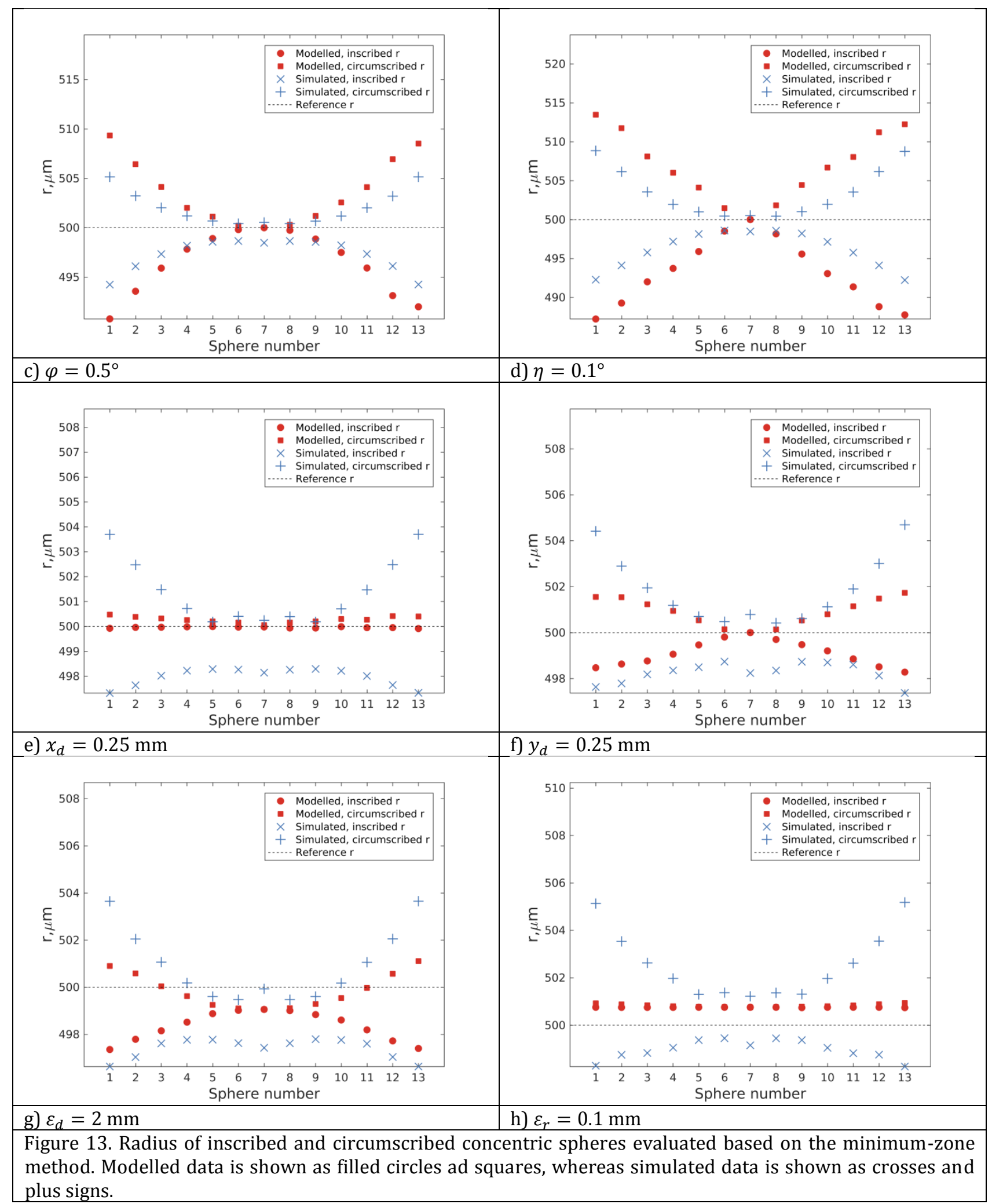

Note, that some error sources, like $\theta, \varepsilon_{d}$ and $\varepsilon_{r}$ cause offset of sphere center point without significantly affecting form error and radius. Therefore one can observe large dispersion of signed distances $d_{i}$ (figures 11, A.1 and A.2) but relatively low error in radius and form error (figure 13). 


\section{Evaluation of the geometrical error model on experimental data}

\subsection{Evaluation object and CT system}

In the following study, we employ the object described in [24], which was used in several studies [11], [25] for estimation of geometrical parameters of a CT system. The object consists of 49 steel spheres with $2.5 \mathrm{~mm}$ diameter arranged along a cylindrical carbon-fiber support, as shown in figure 14. The spheres are numbered 1 to 49 (see figure 15). Sphere number 2 is included to identify the orientation of the object in both radiographic and volumetric data. All sphere center positions except for sphere 2 were calibrated using tactile coordinate measuring machine. We therefore exclude sphere 2 from analysis. The object was measured using a Zeiss Prismo coordinate measuring machine equipped with a rotary table. The maximum permissible error of the coordinate measuring machine is $(2+L / 300) \mu \mathrm{m}$, where $L$ is the measured length in $\mathrm{mm}$ [24]. Variation of sphere diameter and form error is below $0.25 \mu \mathrm{m}$, and surface roughness $R_{a}$ is below $0.5 \mu \mathrm{m}$ [24].

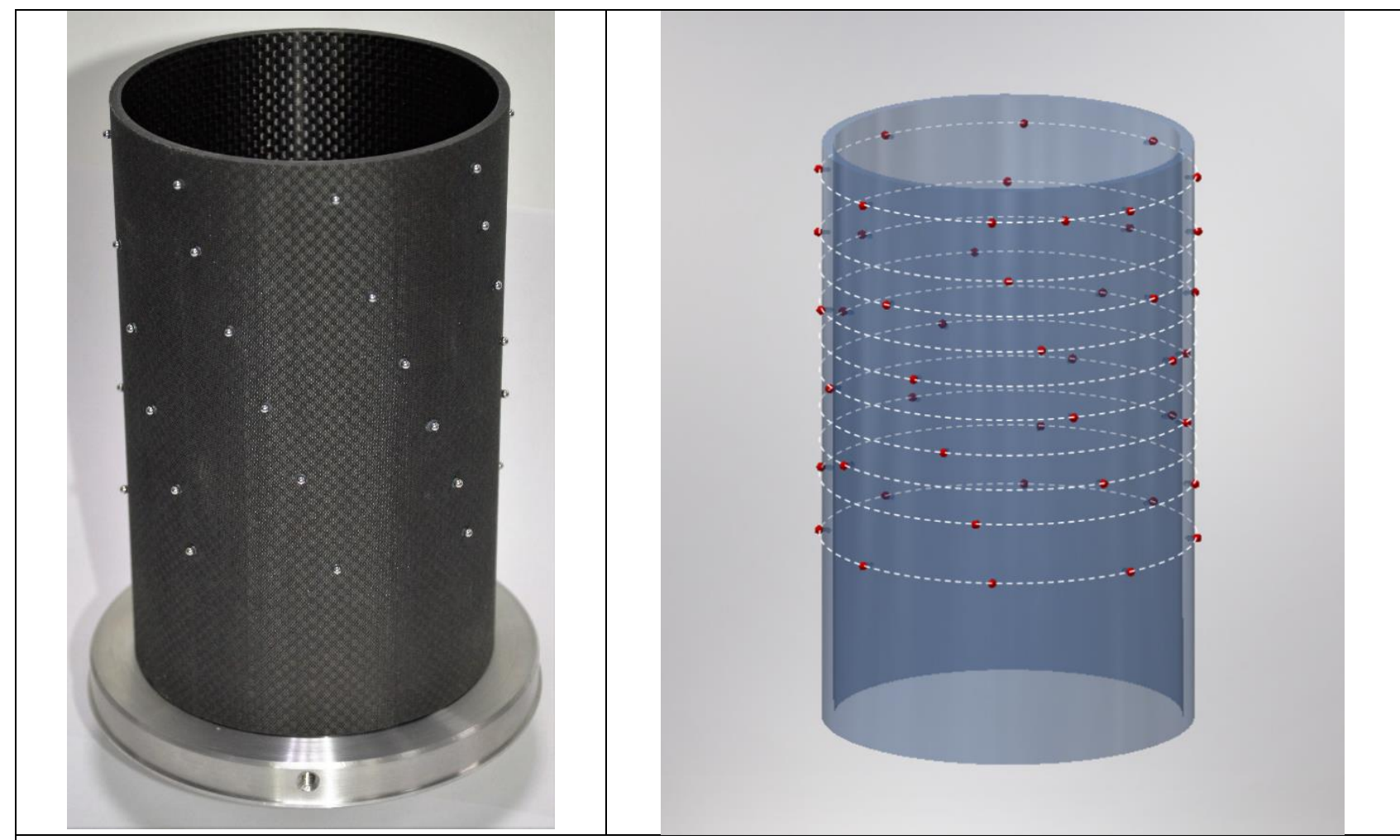

Figure 14. (Left) Photo of the object used in the experimental study. (Right) CAD model of the object used in the experimental study. Spheres are arranged in 10 circular trajectories along the outer circumference of a carbon fiber support. (Figure reproduced with permission from [11]) 


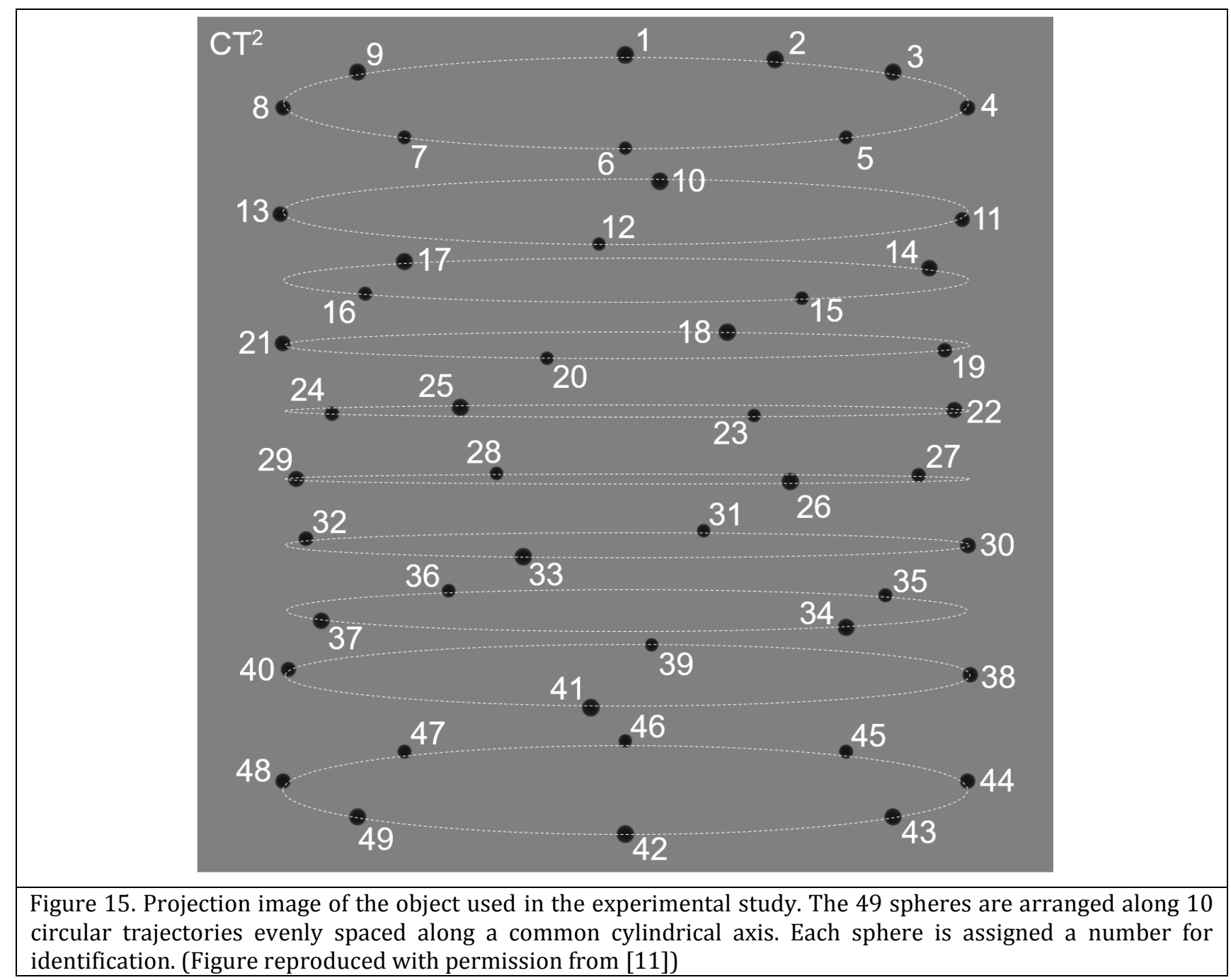

Two sets of radiographs were acquired on an experimental CT system at the Centre of Excellence Telč, Czech Republic. General parameters of the data acquisition system are summarized in table 3. The CT system was intentionally misaligned and the corresponding geometrical parameters, listed in table 4, were estimated using newly developed procedure for measurement of CT system geometry; more information on this procedure can be found in [11]. It should be noted that the procedure in [11] measures not only the geometrical parameters of the CT system, but also the position and orientation of the reference object within the GCF. Consequently, not only distances between the spheres can be used for comparison of experimental and modelled data but also positions of each individual sphere. Both datasets were reconstructed by FDK algorithm with a Ramp filter using Nikon Metrology XT Software Suite with the parameters presented in table 5. No corrections were applied either during or after reconstruction. All surfaces were determined using the local adaptive thresholding method with 4.0 voxels search distance in VGStudioMAX. Initial contour values were selected as the arithmetic mean of grey values corresponding to the carbon-fiber support peak and steel peak in the grey-value histogram. Surfaces were converted to point clouds in VGStudioMAX using built-in "manual" mode with a volumetric sampling interval approximately equal to half of the voxel size $(30 \mu \mathrm{m})$. Here and further, we refer to the resulted point cloud as experimental point cloud.

The settings of the geometrical error model are described in table 4. Parameters $\varepsilon_{r}$ and $\varepsilon_{d}$ were set to 0 . The position of each individual sphere in the GCF was obtained using the procedure described in [11]. The modelled and nominal point clouds were obtained following the same steps as discussed in sections 6.1 and 6.3, respectively 
Table 3. Specification of CT system used in the experimental study.

\begin{tabular}{|l|l|}
\hline Parameter & Value \\
\hline Detector size & $409.6 \times 409.6 \mathrm{~mm}$ \\
\hline Number of pixels & $2048 \times 2048$ \\
\hline Pixel size & $0.2 \mathrm{~mm}$ \\
\hline Detector bit-depth & $15 \mathrm{bit}$ \\
\hline Number of projections & 720 \\
\hline Angular step & $0.5^{\circ}$ \\
\hline
\end{tabular}

Table 4. Geometrical parameters of the CT system used for experimental study as estimated by the procedure described in [11].

\begin{tabular}{|l|l|l|}
\hline Parameter & Dataset 1 & Dataset 2 \\
\hline$z_{r}, \mathrm{~mm}$ & -400.662 & -400.668 \\
\hline$x_{d}, \mathrm{~mm}$ & -0.132 & -0.030 \\
\hline$y_{d}, \mathrm{~mm}$ & 0.122 & 0.436 \\
\hline$z_{d}, \mathrm{~mm}$ & -1212.424 & -1209.897 \\
\hline$\theta,^{\circ}$ & 0.241 & -0.450 \\
\hline$\varphi,^{\circ}$ & 0.074 & 0.097 \\
\hline$\eta,^{\circ}$ & 0.022 & 0.027 \\
\hline
\end{tabular}

Table 5. Reconstruction settings.

\begin{tabular}{|l|l|l|}
\hline Parameter & Dataset 1 & Dataset 2 \\
\hline Source-to-rotation distance, $\mathrm{mm}$ & 400.662 & 400.668 \\
\hline Source-to-detector distance, $\mathrm{mm}$ & 1212.424 & 1209.897 \\
\hline Voxel size, $\mu \mathrm{m}$ & 66.093 & 66.232 \\
\hline Number of voxels & \multicolumn{2}{|c|}{$2048 \times 2048 \times 2048$} \\
\hline Reconstruction filter & \multicolumn{2}{|c|}{ Ramp } \\
\hline
\end{tabular}

\subsection{Experimental results}

In figure 16, left column, we show a grey-value slice of sphere 8 for datasets 1 and 2 . In dataset 1 , double-borders and blur can be observed. Double-borders are less pronounced for dataset 2; however, blur is still evident. Following the same analysis steps as in section 6, we first show the threedimensional rendering of the point cloud representing sphere 8 color-mapped according to the orthogonal distance between internal and external point clouds in order to assess amount of "ghost" material. 


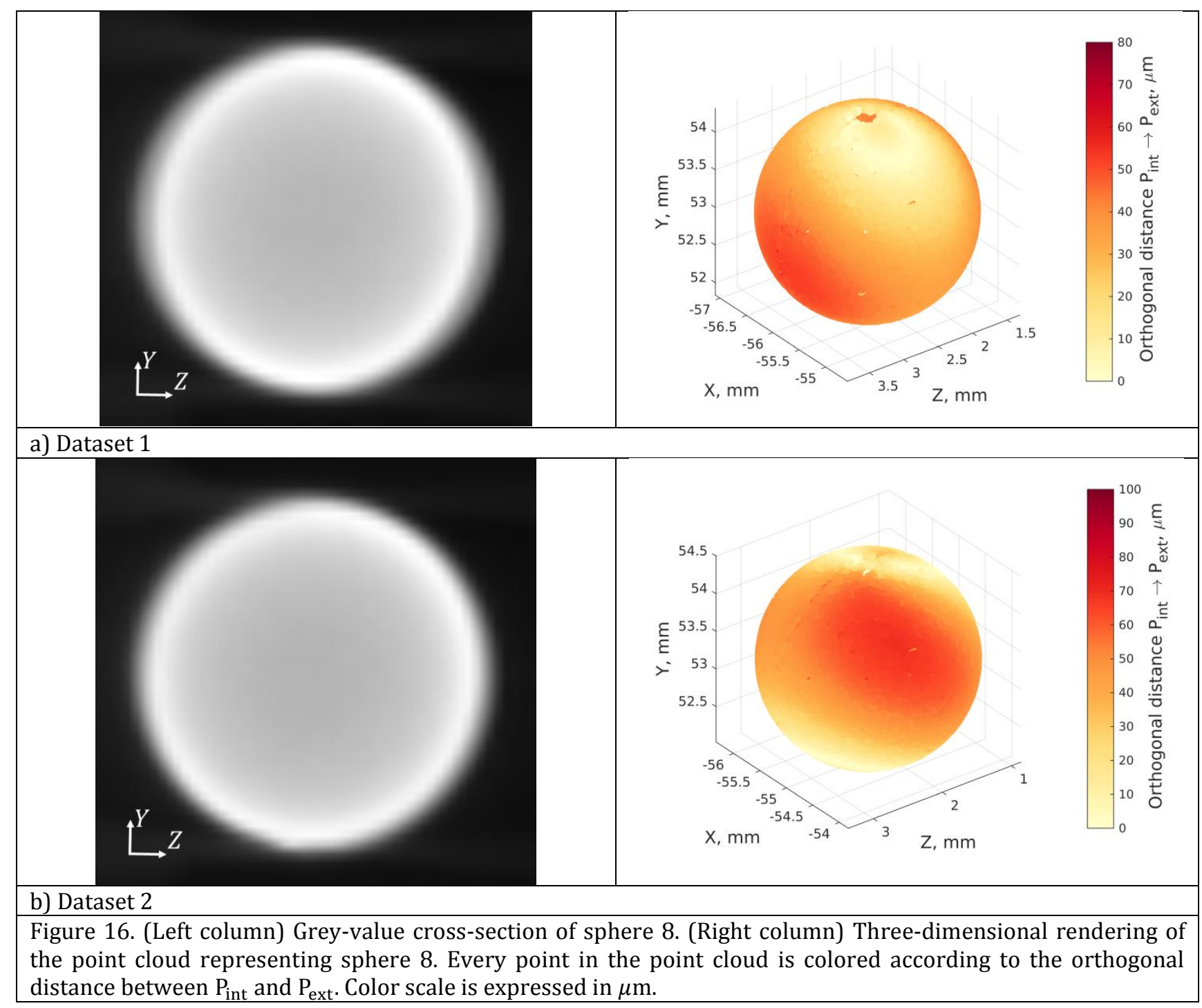

A comparison of both experimental and modelled point cloud against the nominal point cloud is shown in figure 17. The mean value of signed distances for experimental data is positively offset, i.e. reconstructed sphere has bigger diameter than its reference value. This offset can be explained by the influence of beam hardening artifacts on the grey-value profile of the reconstructed volume [25]. In appendix A, figure A.3 we show the 3D rendering of the point cloud corresponding to sphere 8 colormapped to signed distances $d_{i}$ for both datasets. In figure 18, we present summarized statistics for all spheres. The geometrical error model succeeded in predicting the observed behaviors from experimentally-acquired data up to the aforementioned offset.

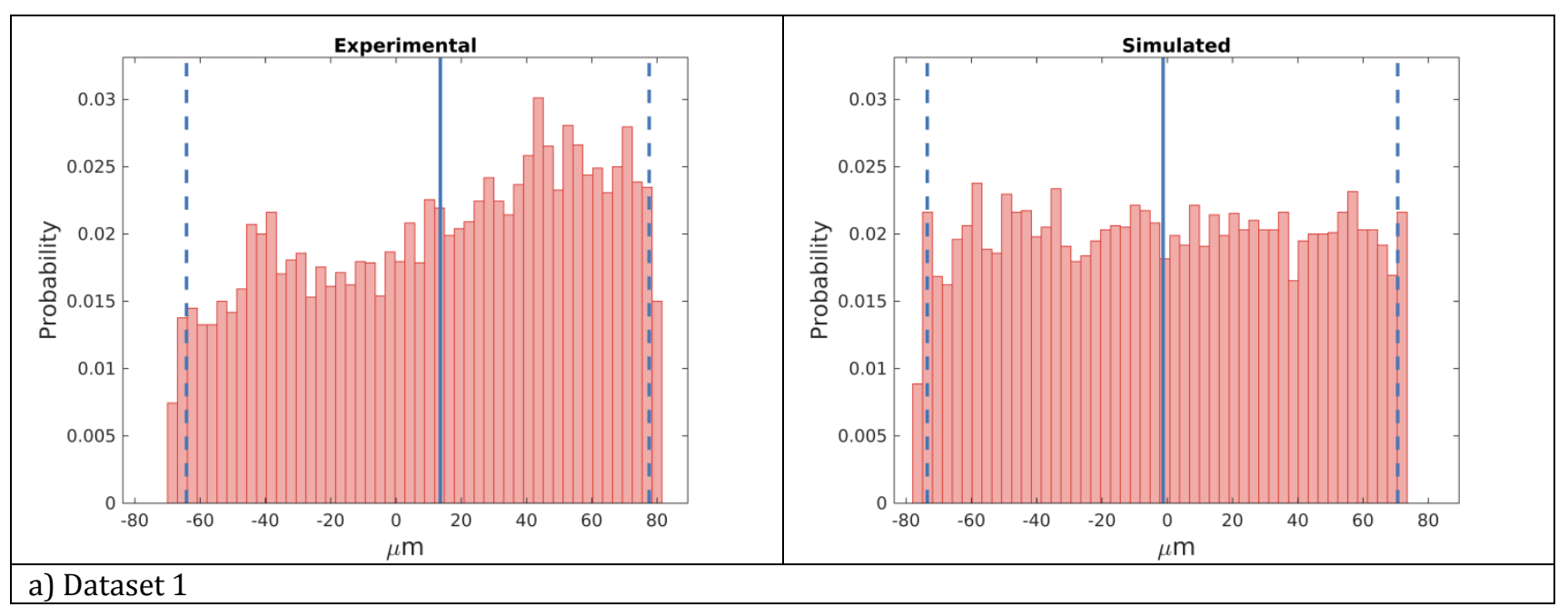



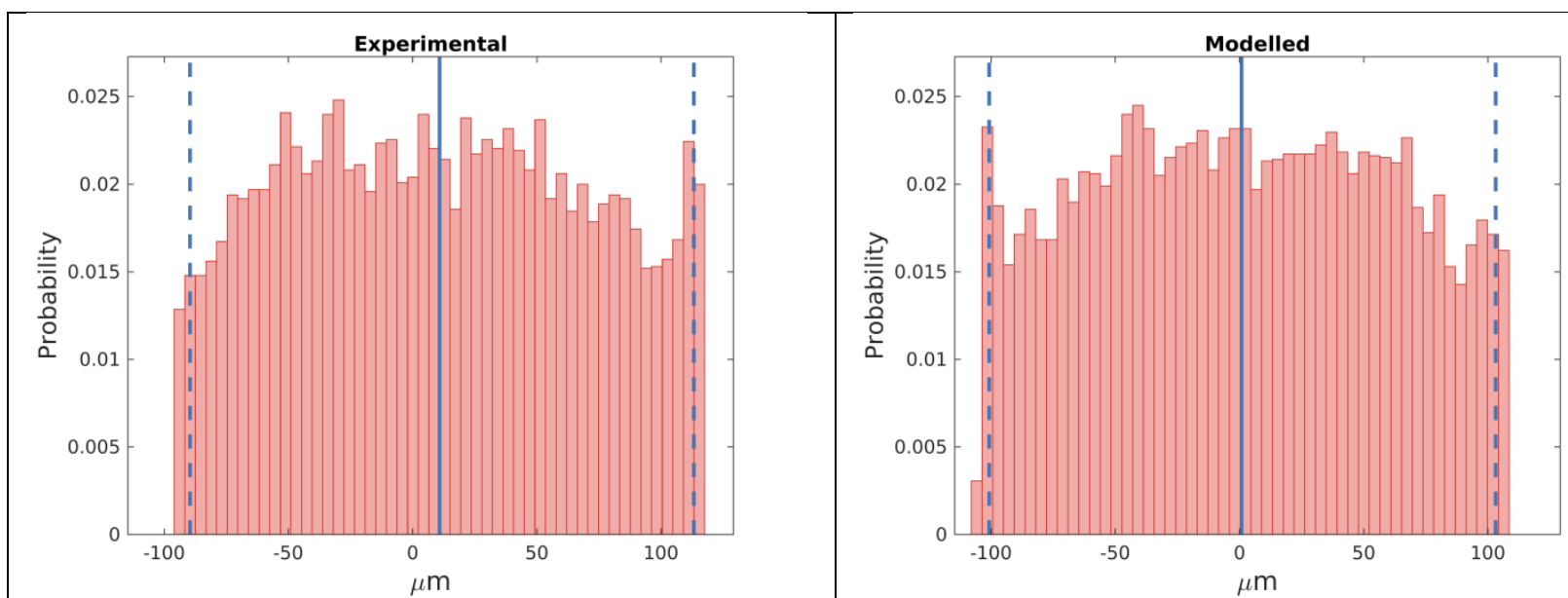

b) Dataset 2

Figure 17. Histogram of signed distances between experimental and nominal point clouds (left column) and between modelled and nominal point clouds (right column) for sphere 8. Mean values and corresponding 95\% confidence interval (based on $2.5 \%$ and $97.5 \%$ quantile) are shown as solid and dashed lines, respectively. Histograms are normalized by probability, i.e. the sum of the bar heights is less than or equal to 1 .
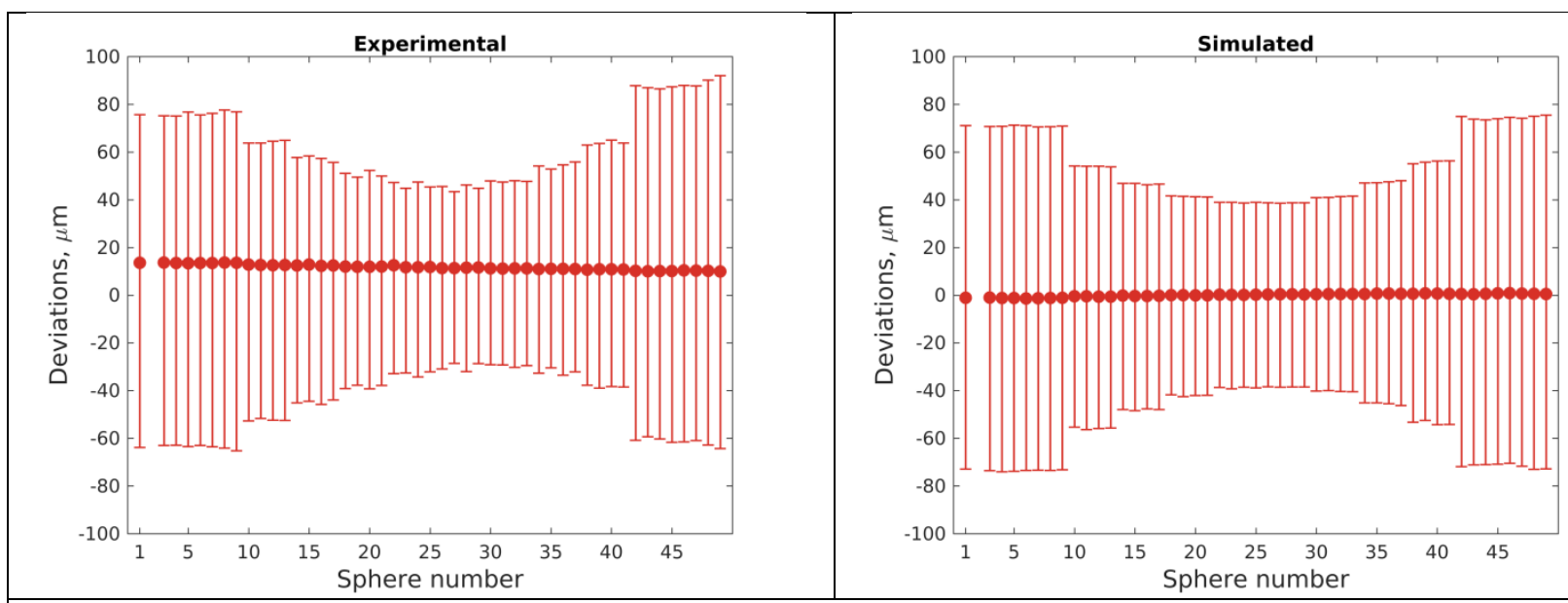

a) Dataset 1
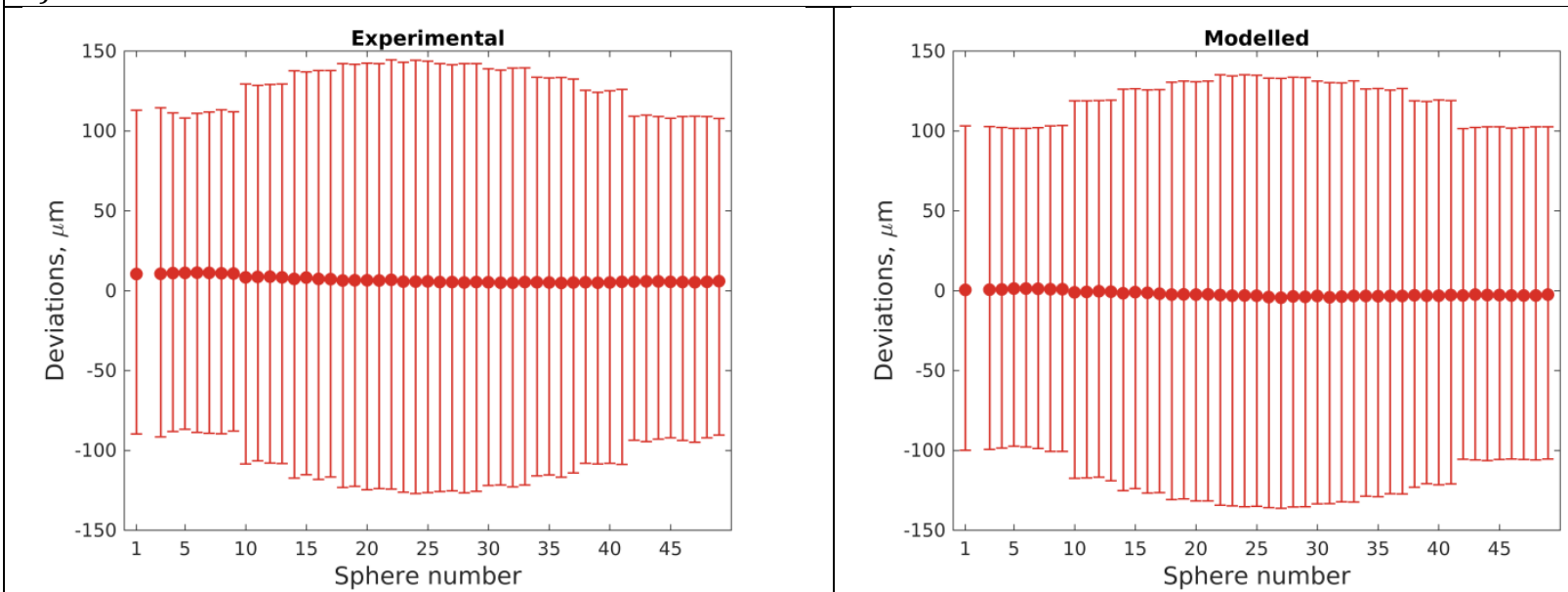

b) Dataset 2

Figure 18. Signed distance between experimental and nominal point clouds (left column), and modelled and nominal point clouds (right column) for individual spheres. Mean is shown as filled red circles. Bottom and top limit of error bar correspond to the $2.5 \%$ and $97.5 \%$ quantiles, respectively.

Finally, figure 19 compares dimensional measurement results performed on experimental and modelled data. Following the same steps as in the simulation study (section 7), center point position of each sphere is measured using least squares fit (figure 19a-d), whereas form error is assessed using minimum-zone method (figure 19e). The significant offset of the detector center point along $Y$ axis (table 4) resulted in a shift of the sphere center points from their expected positions. These effects are 
noted as large dispersions of signed distances $d_{i}$ (figures 17, 18 and A3), albeit relatively low radius and form error (figure 19e). The geometrical error model is capable of reproducing the influence of the misalignments on the location of the sphere center point, as demonstrated in figure 19a-d. Measured inscribed and circumscribed radii on modelled and experimental data, shown in figure 19e, are offset similarly to the data shown in figure 18. We conclude that the geometrical error model is capable of reproducing the influence of geometrical misalignments on the dimensional measurement results.

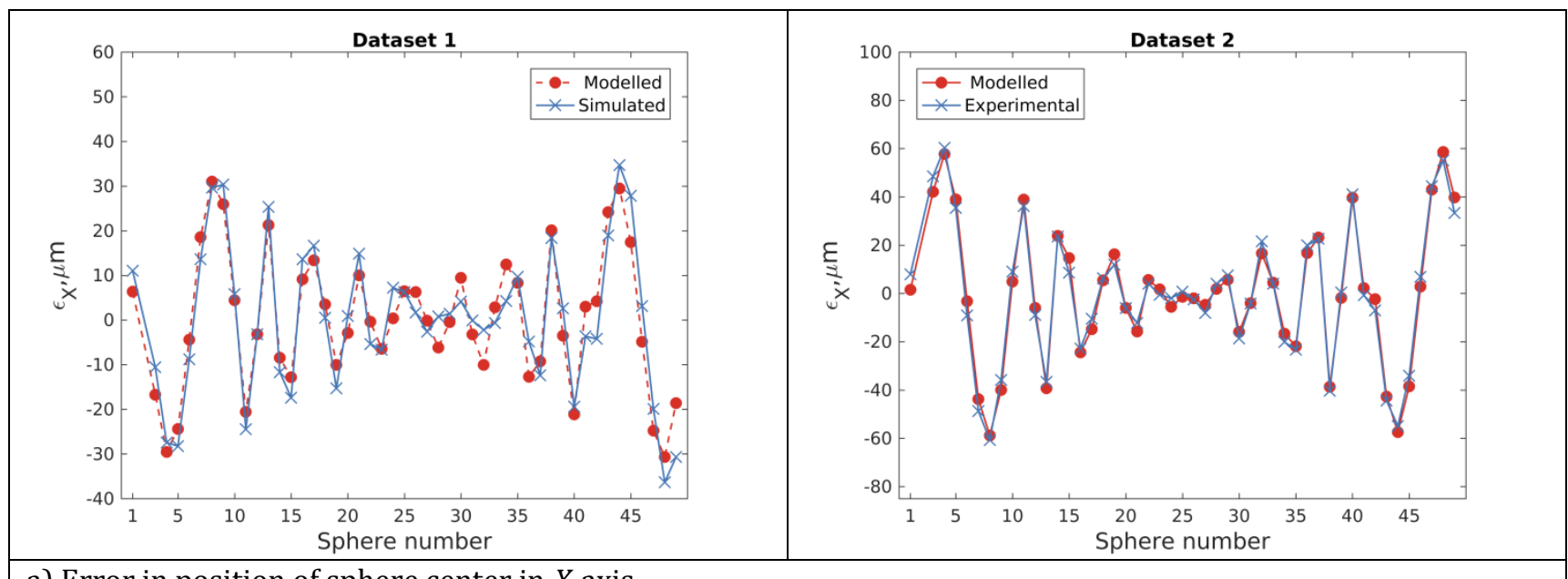

a) Error in position of sphere center in $X$ axis.
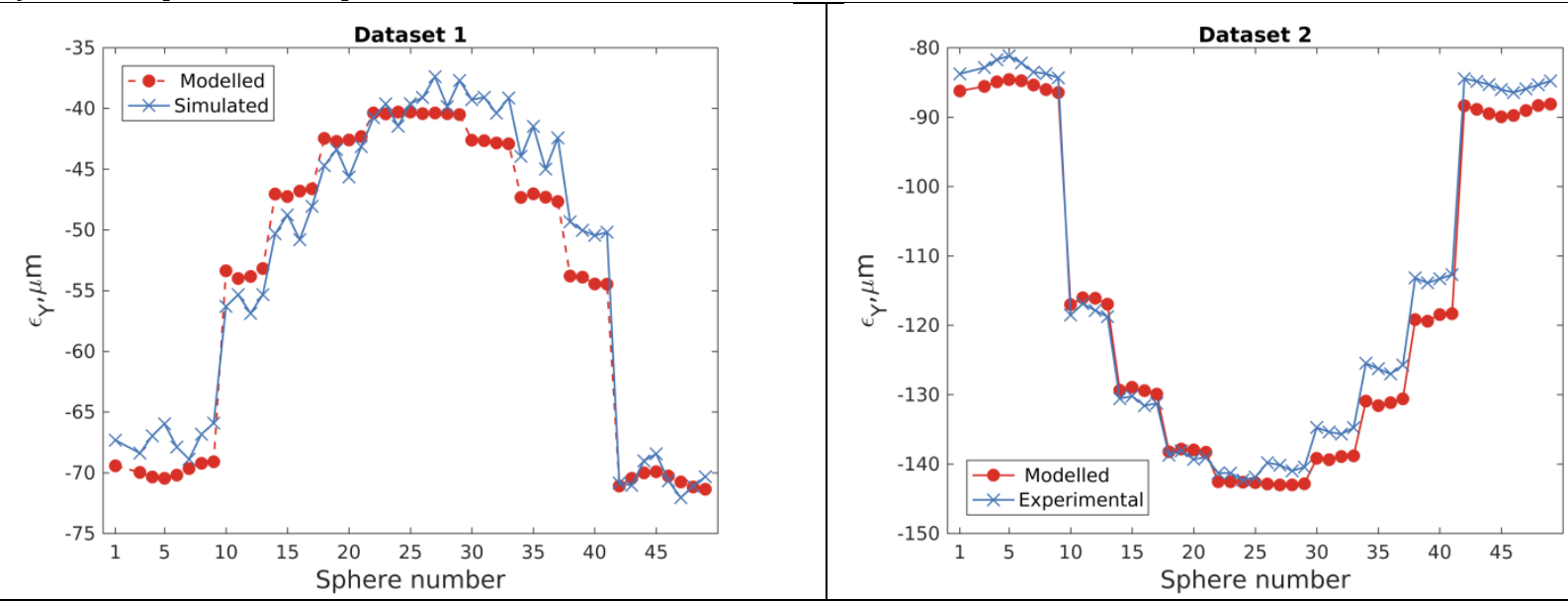

b) Error in position of sphere center in $Y$ axis.
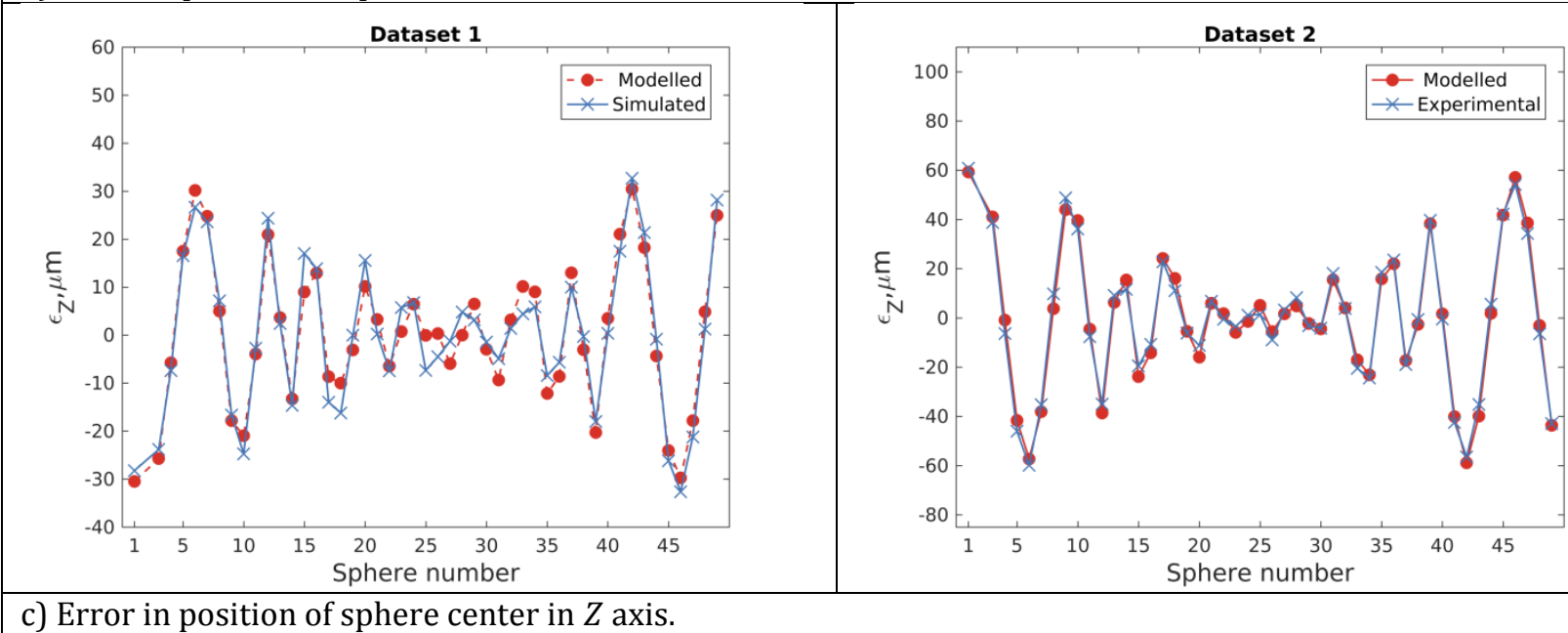

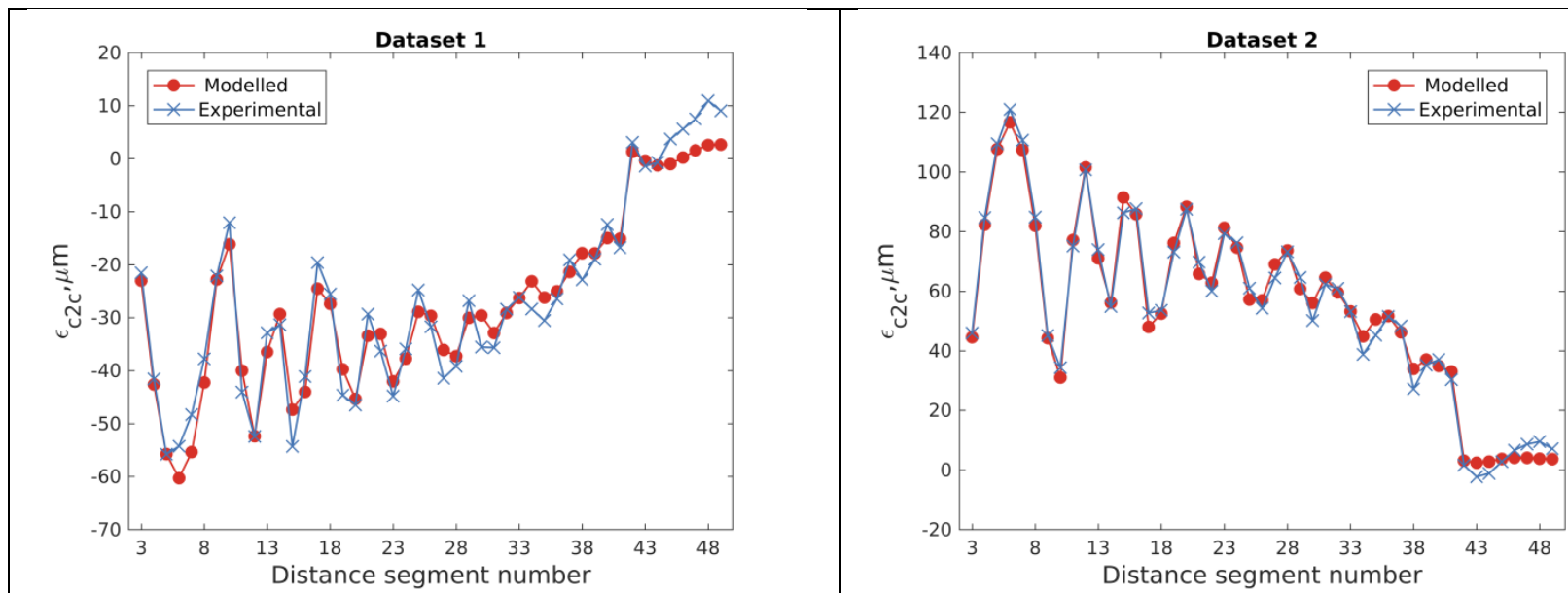

d) Absolute error in center-to-center distance from sphere 1 to all consecutive spheres (3-49), i.e. point 3 corresponds to the distance between sphere 1 and 3. Modelled data is shown as solid lines with filled circles, whereas experimental data is shown as dashed lines and crosses.
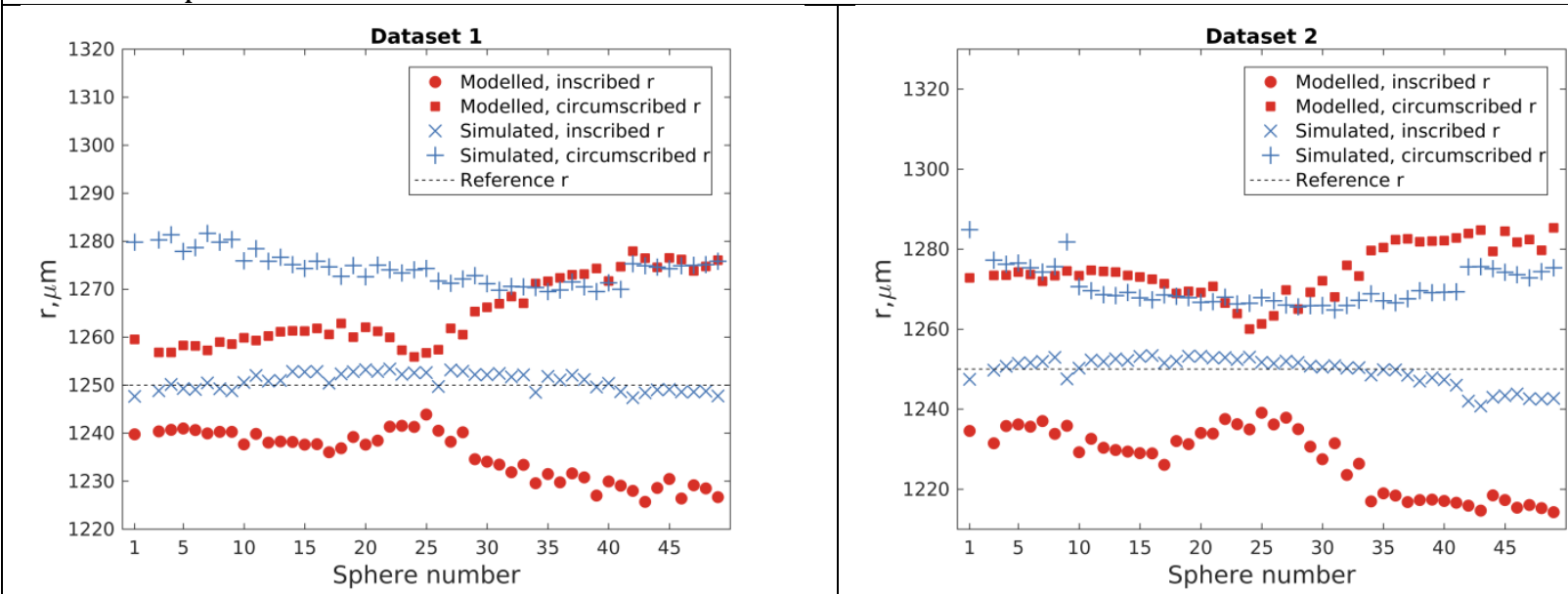

e) Radii of inscribed and circumscribed concentric spheres. Modelled data is shown as filled circles and squares, whereas experimental data is shown as crosses and plus sign.

Figure 19. Dimensional errors in experimental datasets and dimensional errors predicted by the proposed geometrical error model. The left column presents dimensional measurements for dataset 1 , whereas the right column corresponds to dataset 2. Center point position of each sphere is measured using least squares fit, whereas form error is assessed using minimum-zone method. Sphere 2 was excluded from comparison.

\section{Conclusion}

Assessment of uncertainty in CT dimensional measurements is fundamental for acceptance of CT as a measuring instrument. A feasible method for quantification of measurement uncertainty is by means of the Monte Carlo by simulation method. However, the computational cost of simulating the CT measurement procedure prohibits the practical application of Monte Carlo methods to real measurement tasks. In this paper we introduce a geometrical error model as an alternative to the computationally-demanding Monte Carlo by simulation method of uncertainty assessment.

The geometrical error model is based on the forward- and back-projection of an object's surface points extracted from CAD-model to assess discrepancies in the radiographic image coordinates assigned to the projected edges between an aligned system and a system with misalignments. We found that the proposed geometrical error model is successful in capturing errors in the tomographically reconstructed edges from simulated and experimental datasets of a simple object consisting of spheres in a particular arrangement. The validity of the model is also evaluated by comparing dimensional measurements (sphere center-to-center distances and radii) performed on simulated and experimental data. Extension of the proposed method to other geometrical error parameters and objects is a topic of future investigation.

The computational load associated with the implementation of the proposed geometrical error model, namely the procedure for forward- and back-projection of surface points, is only a fraction of 
what is required for full simulation of the CT measurement procedure. Simulation time of the CT measurement procedure depends on many parameters, such as hardware, implementation strategy, dataset size, parameters set, etc. Typically cited simulation times are between a few dozens of minutes up to several hours for a single simulation run. A single run of the proposed geometrical error model (MATLAB implementation, Intel Core i7 (5th Gen) 5600U / $2.6 \mathrm{GHz}$ ) for the evaluation object described in section 6.1 took approximately 1 second including application of Gaussian sphere fit to every sphere. Since the algorithm used to implement the proposed method processes every surface point separately, it is well suited for parallel processing. The proposed method therefore provides a considerable reduction in computation time in a single Monte Carlo run.

\section{Acknowledgments}

The research performed for this publication was funded by the European Union 7th Framework Programme under grant agreement 607817. The authors would like to thank Petr Heřmanek, Michal Vopalensky, Ivana Kumpova, and Daniel Vavřik for their help and support during the acquisition of experimental data at Centre of Excellence Telč. Additionally, the authors greatly acknowledge Dr. Michael McCarthy for his support at the National Physical Laboratory. 


\section{References}

1. Ferrucci, M., Leach, R.K., Giusca, C., Carmignato, S. and Dewulf, W., 2015. Towards geometrical calibration of x-ray computed tomography systems - a review. Measurement Science and Technology, 26(9), p.092003.

2. Ferrucci, M., Ametova, E., Carmignato, S. and Dewulf, W., 2016. Evaluating the effects of detector angular misalignments on simulated computed tomography data. Precision Engineering, 45, pp.230-241.

3. Evaluation of measurement data - guide for the expression of uncertainty in measurement. JCGM 100: 2008

4. Kruth, J.P., Bartscher, M., Carmignato, S., Schmitt, R., De Chiffre, L. and Weckenmann, A., 2011. Computed tomography for dimensional metrology. CIRP Annals-Manufacturing Technology, 60(2), pp.821-842.

5. Hiller, J. and Reindl, L.M., 2012. A computer simulation platform for the estimation of measurement uncertainties in dimensional X-ray computed tomography. Measurement, 45(8), pp.2166-2182.

6. Helmecke, E., Fleßner, M., Kaufmann, M., Staude, A. and Hausotte, T., 2016. Numerical measurement uncertainty determination for dimensional measurements of microparts with CT. In 6th Conference on Industrial Computed Tomography, Wels, Austria (iCT 2016).

7. Rossi, D. and Willsky, A., 1984. Reconstruction from projections based on detection and estimation of objects--Parts I and II: Performance analysis and robustness analysis. IEEE Transactions on Acoustics, Speech, and Signal Processing, 32(4), pp.886-906.

8. Soussen, C. and Mohammad-Djafari, A., 2004. Polygonal and polyhedral contour reconstruction in computed tomography. IEEE Transactions on Image Processing, 13(11), pp.1507-1523.

9. Baumgart, B.G., 1974. Geometric modeling for computer vision (No. STAN-CS-74-463). Stanford Univ CA Deptment of Computer Science.

10. Yang, K., Kwan, A.L., Miller, D.F. and Boone, J.M., 2006. A geometric calibration method for cone beam CT systems. Medical physics, 33(6), pp.1695-1706.

11. Ferrucci M., Hermanek P., Ametova E., Carmignato, S., Dewulf W., 2018 Measurement of the X-ray computed tomography instrument geometry by minimization of reprojection errors-implementation on simulated data Submitted to Precision Engineering.

12. Pan, X., Sidky, E.Y. and Vannier, M., 2009. Why do commercial CT scanners still employ traditional, filtered back-projection for image reconstruction?. Inverse problems, 25(12), p.123009.

13. Tuy, H.K., 1983. An inversion formula for cone-beam reconstruction. SIAM Journal on Applied Mathematics, 43(3), pp.546-552.

14. Ametova, E., Ferrucci, M. and Dewulf, W., 2017, February. A tool for reducing cone-beam artifacts in computed tomography data. In The e-Journal of Nondestructive Testing (No. 2017-03).

15. VDI/VDE 2630 - Part 1.3 Computed tomography in dimensional measurement, Guideline for the application of DIN EN ISO 10360 for coordinate measuring machines with CT sensors 12/2011

16. Hartley, R. and Zisserman, A., 2003. Multiple view geometry in computer vision. Cambridge university press. ISBN: 0521540518.

17. Solomon, C. and Breckon, T., 2011. Fundamentals of Digital Image Processing: A practical approach with examples in Matlab. John Wiley \& Sons. ISBN: 978-0-470-84472-4.

18. Woop, S., Feng, L., Wald, I. and Benthin, C., 2013, July. Embree ray tracing kernels for CPUs and the Xeon Phi architecture. In ACM SIGGRAPH 2013 Talks (p. 44). ACM.

19. Muller, M.E., 1959. A note on a method for generating points uniformly on n-dimensional spheres. Communications of the ACM, 2(4), pp.19-20.

20. Duda, R.O., Hart, P.E. and Stork, D.G., 2000. Pattern classification. John Wiley \& Sons. ISBN: 978-0-47105669-0.

21. Taubin, G., Cukierman, F., Sullivan, S., Ponce, J. and Kriegman, D.J., 1992, June. Parametrizing and fitting bounded algebraic curves and surfaces. In Computer Vision and Pattern Recognition, 1992. Proceedings CVPR'92., 1992 IEEE Computer Society Conference on (pp. 103-108). IEEE.

22. Schneider, P. and Eberly, D.H., 2002. Geometric tools for computer graphics. Morgan Kaufmann. ISBN: 9781558605947.

23. Muralikrishnan, B. and Raja, J., 2008. Computational surface and roundness metrology. Springer Science \& Business Media. ISBN: 978-1-84800-297-5

24. Hermanek, P., Ferrucci, M., Carmignato, S. and Dewulf, W., 2017. Optimized reference object for assessment of computed tomography instrument geometry. In The e-Journal of Nondestructive Testing (No. 2017-03).

25. Tan, Y., Kiekens, K., Kruth, J.P., Voet, A., Dewulf, W., 2011. Material Dependent Thresholding for Dimensional X-ray Computed Tomography. International Symposium on Digital Industrial Radiology and Computed Tomography, Berlin, Germany 
Appendix A

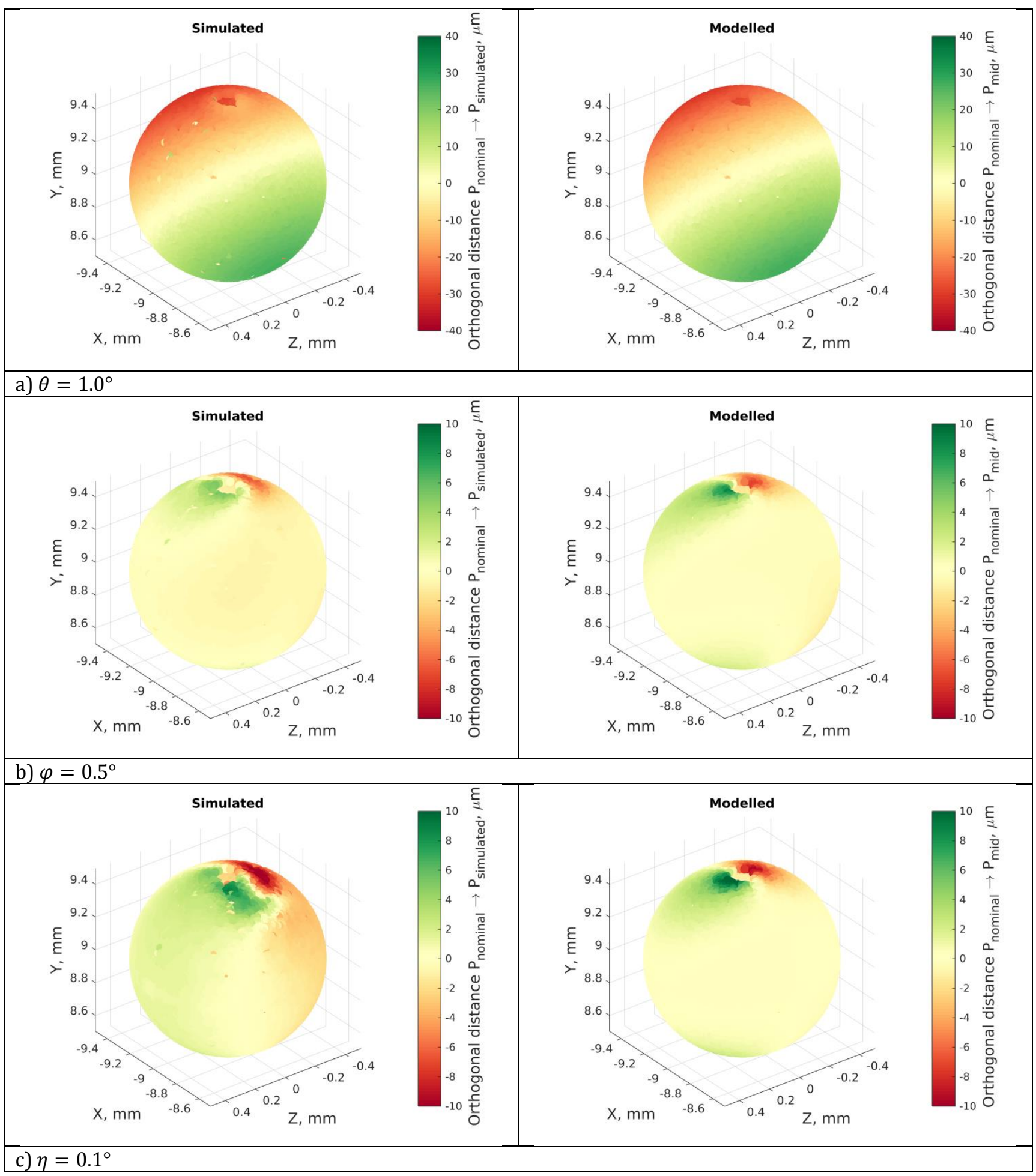




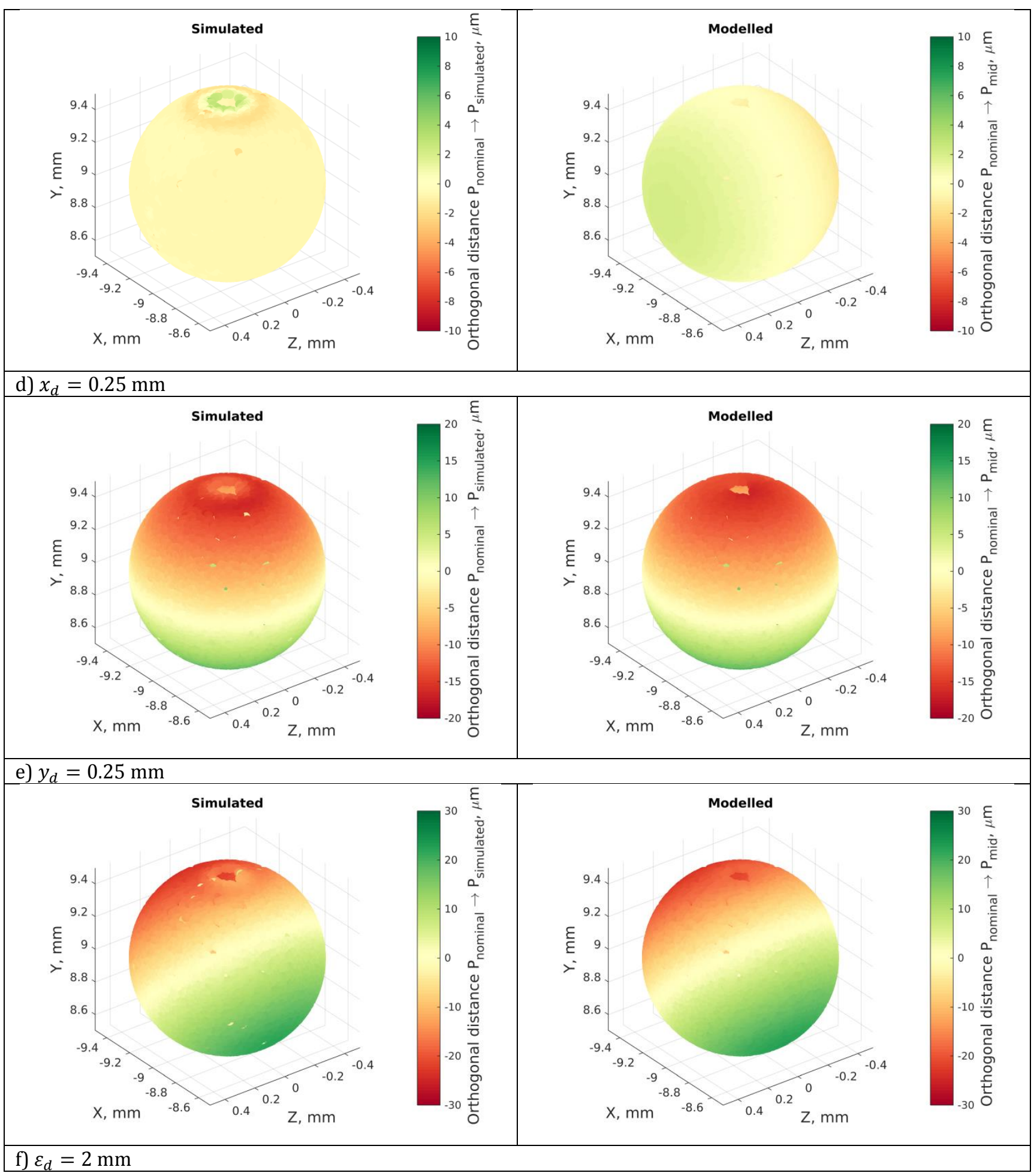



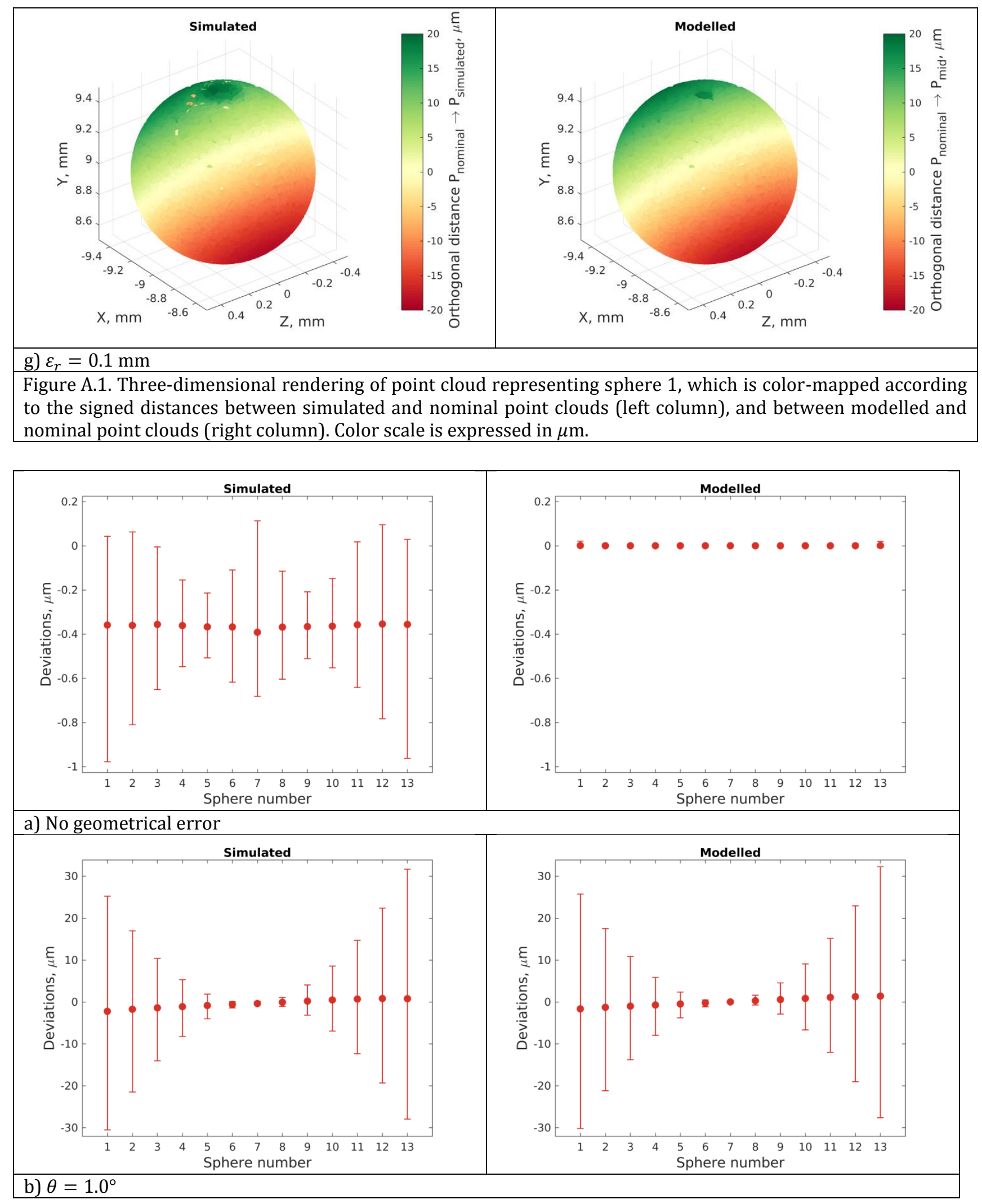


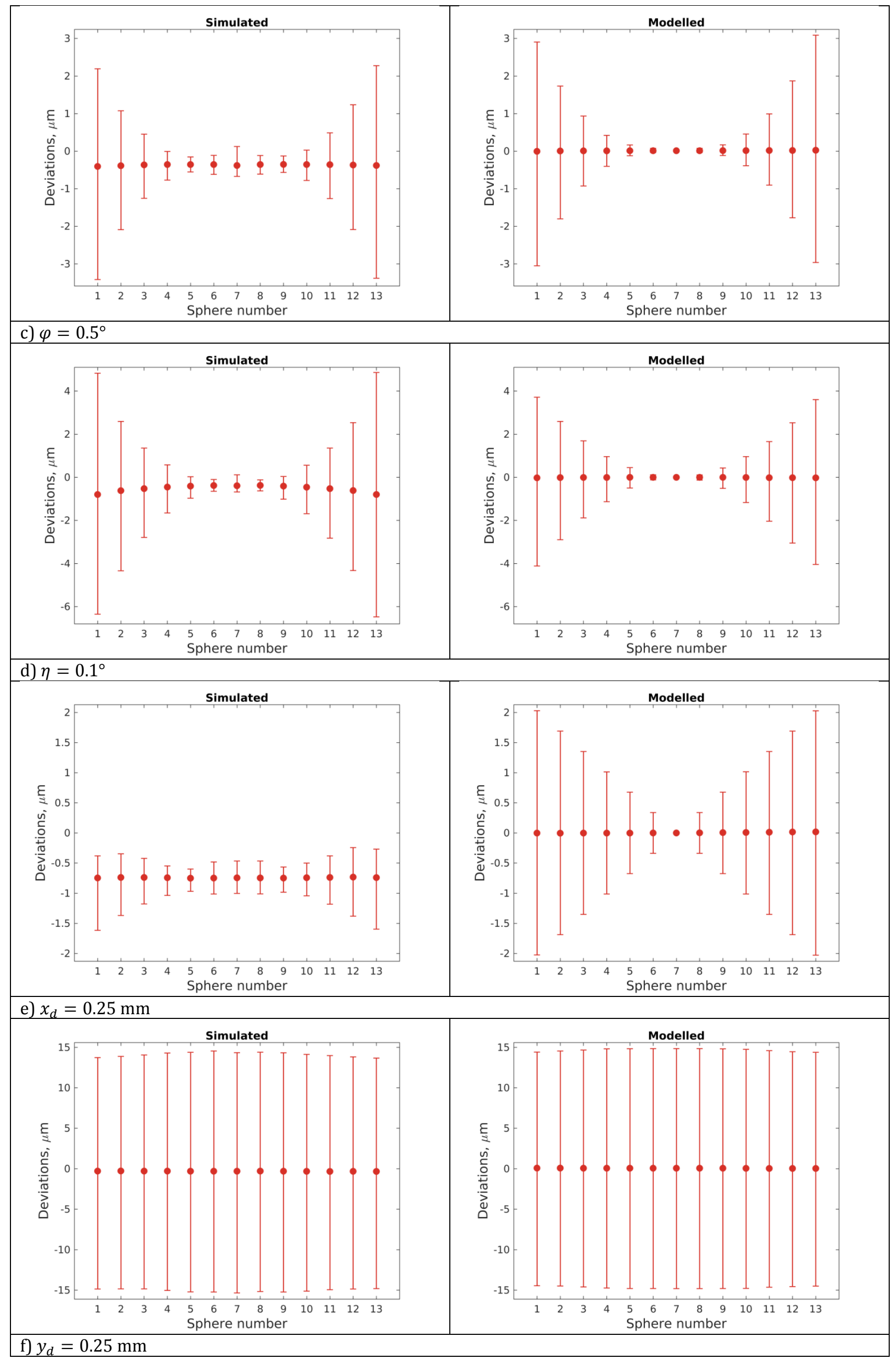




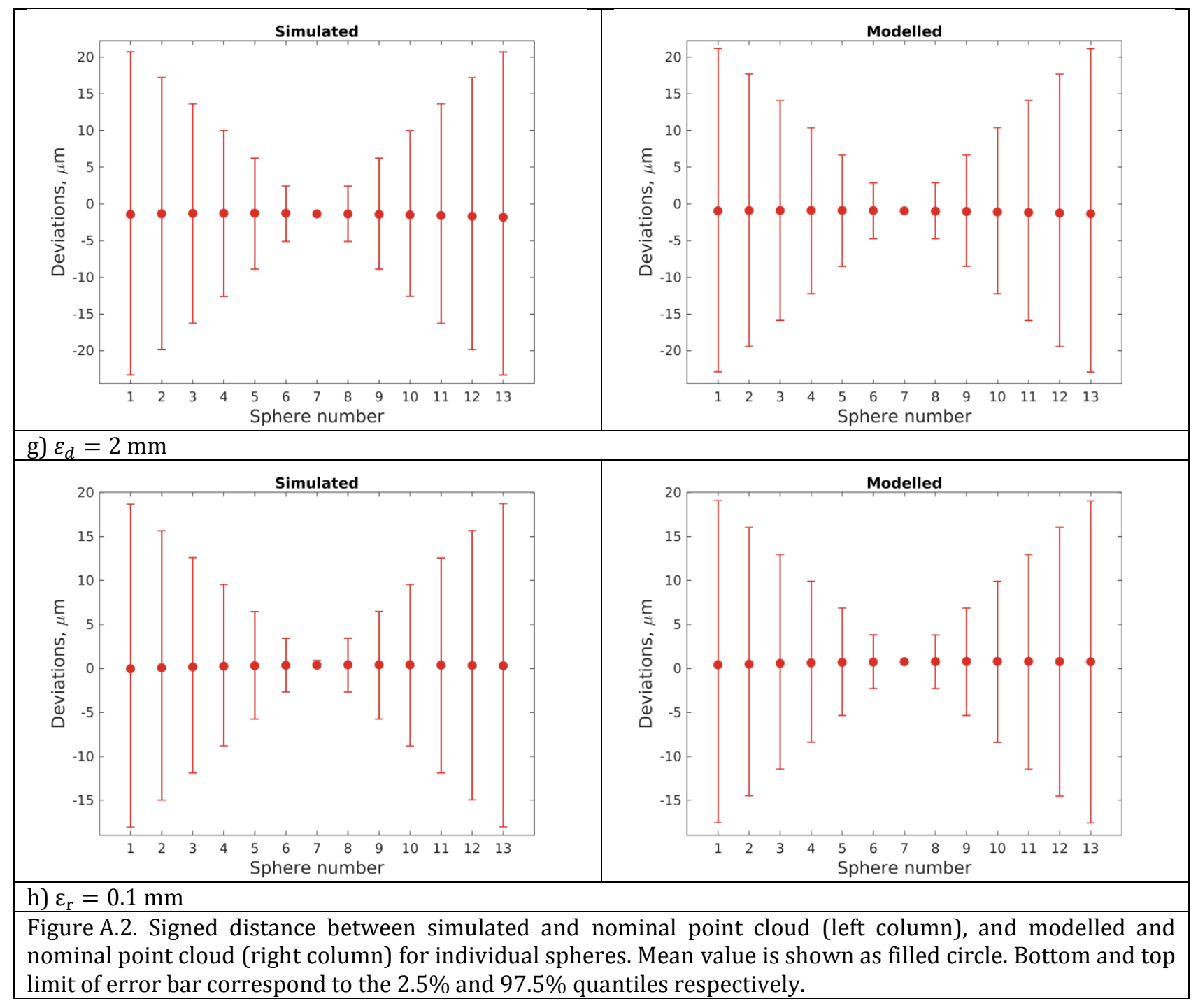




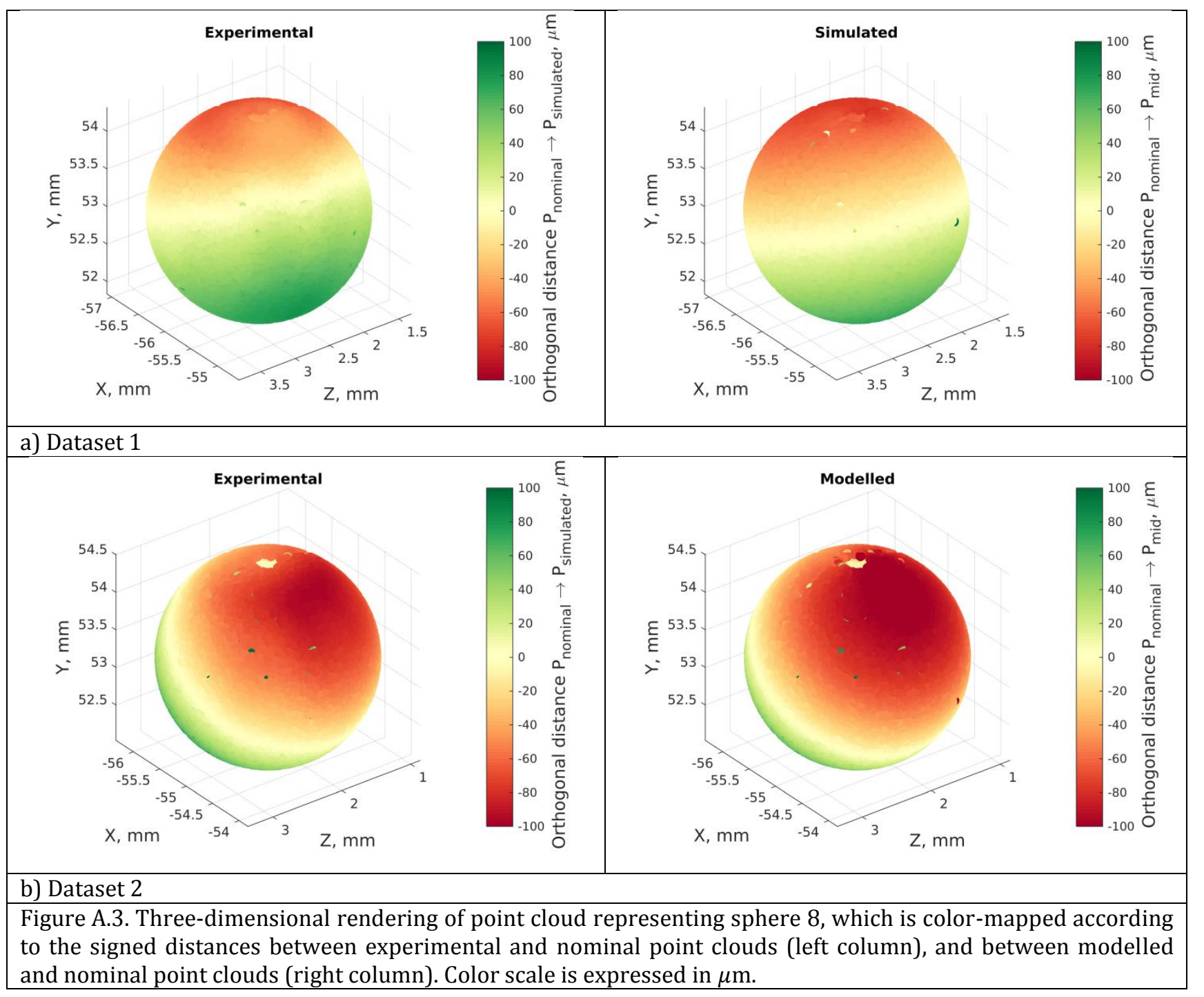

